
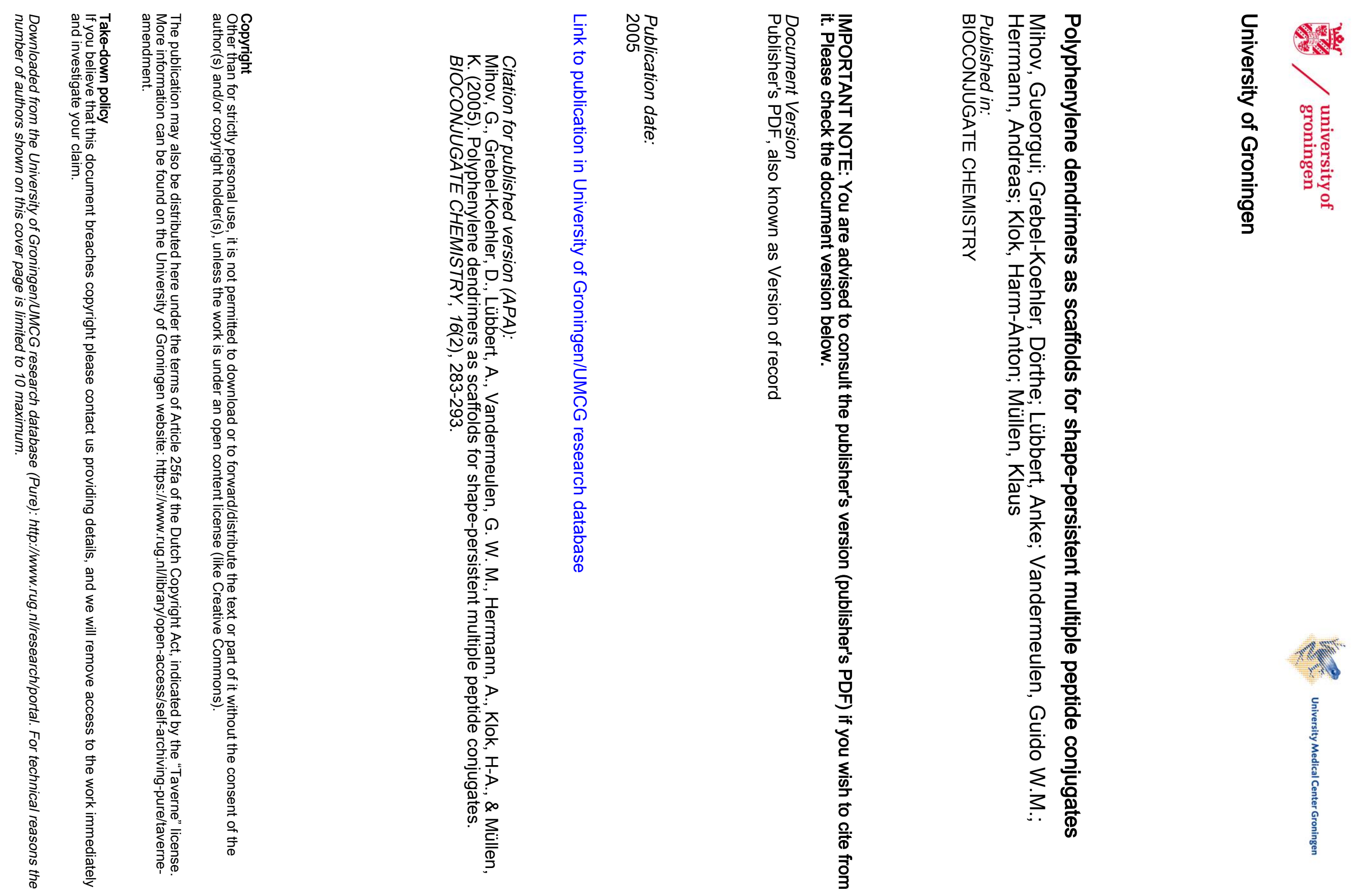


\title{
Polyphenylene Dendrimers as Scaffolds for Shape-Persistent Multiple Peptide Conjugates
}

\author{
Gueorgui Mihov, ${ }^{\dagger}$ Dörthe Grebel-Koehler, ${ }^{\dagger}$ Anke Lübbert, ${ }^{\dagger}$ Guido W. M. Vandermeulen, ${ }^{\dagger}$ \\ Andreas Herrmann, ${ }^{\dagger}$ Harm-Anton Klok, ${ }^{\ddagger}$ and Klaus Müllen ${ }^{* \dagger}$
}

Max Planck Institute for Polymer Research Ackermannweg 10D-55128 Mainz, Germany, and Ecole Polytechnique Fédérale de Lausanne (EPFL) Institut des Matériaux, Laboratoire des Polymères Bâtiment MX-D, CH 1015 Lausanne, Switzerland. Received July 8, 2004; Revised Manuscript Received February 2, 2005

\begin{abstract}
The present work describes synthetic concepts for the coupling of peptides to polyphenylene dendrimers (PPDs). Novel functionalized cyclopentadienones have been synthesized whose Diels-Alder cycloaddition with various core molecules leads to polyphenylene dendrimers possessing (protected) amino or carboxyl groups. In addition, the resulting functionalized molecules exhibit the characteristic shapepersistence and monodispersity of PPDs. Their functions have been used for the attachment of polylysine to the dendritic scaffold. Three different methods for the decoration of dendrimers with polypeptides are presented. First, polylysine segments are grafted from the surface of the dendrimers employing $\alpha$-amino acid $N$-carboxyanhydride (NCA) polymerization. Second, the $C$-terminal carboxyl groups of protected polypeptides are activated and then coupled to the amino groups on the surface of the PPD. Finally, cysteine terminated, unprotected peptide sequences are attached to polyphenylene dendrimers utilizing the addition of the sulfhydryl group of a cysteine to the maleimide functions on the dendrimer surface. Moreover, Diels-Alder cycloaddition of suitably functionalized cyclopentadienons to a desymmetized core molecule allows the design of a dendritic scaffold with a specific number of different anchor groups on its periphery. These approaches are important for the tailoring of new, shape-persistent, polyfunctional multiple antigen conjugates.
\end{abstract}

\section{INTRODUCTION}

Dendrimers are monodisperse, highly branched macromolecules $(1-3)$. Their synthesis involves either a convergent or divergent protocol, both implicating the creation of a core molecule and an iterative procedure for the construction of the dendrimer. The high number of functional groups and the well-defined structure make dendrimers attractive carriers for the design of nanoparticles with potential uses in clinical and diagnostic applications. Recent advances in the biomedical applications of various dendritic structures are reviewed by Stiriba et al. (4). The polyphenylene dendrimers (PPDs) ${ }^{1}$ described here are unique shape-persistent and polyfunctional molecules bearing topologically isolated, noninterfering functional groups (5). This is essential for the

* To whom correspondence should be addressed. Prof. Dr. Klaus Müllen, Max Planck Institute for Polymer Research, Ackermannweg 10, D-55128 Mainz, Germany. Phone: +49 (0) 6131 379150/151. Fax: +49 (0) 6131 379350. E-mail: muellen@ mpip-mainz.mpg.de.

$\dagger$ Max Planck Institute for Polymer Research.

Ecole Polytechnique Fédérale de Lausanne (EPFL) Institut des Matériaux.

${ }^{1}$ Abbreviations: PPD, polyphenylene dendrimers; NCA, $\mathrm{N}$ carboxyanhydride; MAP, multiple antigen peptides; PDI, perylenediimide; GPC, gel permeation chromatography; HBTU, $O$-benzotriazole- $N, N, N^{\prime}, N^{\prime}$-tertramethyluronium hexafluorophosphate; HOBt, $N$-hydroxybenzotriazole; DCM, dichloromethane; EDC, $N$-(3-dimethylaminopropyl)- $N$ '-ethylcarbodiimide hydrochloride; DIPEA, diisopropylethylamine; NMWLs, nominal molecular weight limits; PTFE, polytetrafluorethylene; $M_{\mathrm{w}}$, weight-average molecular weight; $M_{\mathrm{n}}$, number- average molecular weight; TFE, 2,2,2-trifluoroethanol; $[\Theta]_{222}$, mean residue elipticity at $222 \mathrm{~nm}$; GnTd, n-generation dendrimer with tetrahedral core; GnPDI, n-generation dendrimer with PDI core. active function of PPDs decorated with short peptide sequences because the bioactivity of the latter is strongly influenced by inter- and intramolecular chain interactions. The attachment of a defined number of oligopeptides onto PPDs has been inspired by the recent achievement of multiple antigen peptides (MAPs) (6).

MAPs are nanoparticles, which, when introduced to an organism, are able to trigger an immune response. In nature, the recognition of foreign bodies (antigens) by antibodies proceeds via the so-called key and lock principle, in which the shape of the antibodies' receptors is complementary to a given pattern on the surface of the antigen that is to be recognized. Complex antigens have a variety of epitopes on different parts of their surface, each of which can be recognized separately by a different antibody. Antigens of special interest are so-called immunogens that trigger an immune response, i.e., the production of antibodies.

Very often, the identifiable part for the antibody is a polypeptide segment. The attachment of several recognizable polypeptides to a nanoparticle is referred to as a MAP. These structures have proven to exhibit amplified immunogenicity and pave the way toward fully synthetic vaccines (7). Currently, MAPs are constructed by attaching the desired polypeptides to a dendritic matrix composed, most frequently, of branched polylysine. It has been shown that a stronger immunogenic effect may be achieved when the MAP system contains two different antigen peptides (8).

However, the oligolysine carriers do not possess a defined spatial structure, which is believed to have an unfavorable effect on their function (6). In fact, inter- and intramolecular interactions of the antigens may modify their secondary structure as well as limit their acces- 
sibility. Therefore, the synthesis of nanosized peptide carriers comprising a well-defined structure and shapepersistence may provide improved MAPs. In this respect, the polyphenylene dendrimers are very promising templates due to their structural perfection and precise spatial definition of functional units in the center, in the dendritic scaffold, or at the periphery. Furthermore, they are chemically inert under the conditions of living organisms, which is another important prerequisite for biologically oriented applications.

In addition, the polyphenylene dendrimers can be synthesized not only as all-carbon structures but also using a strongly fluorescent perylenediimide core (PDI). Recently, we have described several synthetic approaches toward water-soluble and fluorescent PDI chromophores (9). The chromophore adds a further important property, namely, the possibility of facile optical detection of the dendritic conjugate, and such molecules may find diagnostic application. Thus, the aim of the present work is the development of reliable and expandable synthetic routes affording peptide-functionalized polyphenylene dendrimers with prospective application for attachment of antigen peptides onto a shape-persistent, polyphenylene scaffold. A further development is the synthesis of polyphenylene dendrimers bearing two different polypeptides. While we have recently described routes toward the synthesis of novel polyphenylene dendrimers with lysine and glutamic acid moieties anchored on the surface (10), we herein extend this idea by employing different synthetic approaches for the preparation of peptide-PPD conjugates. Polyphenylene dendrimers were functionalized with polylysine following three parallel routes. First, oligolysines were grafted directly from the surface of polyphenylene dendrimers employing $\alpha$-amino acid $N$-carboxyanhydride (NCA) polymerization. Second, protected pentalysine moieties were coupled to PPDs via carbodiimide activation. Third, unprotected hexapeptides, composed of five lysine residues and a $C$-terminal cysteine, were specifically attached to the polyphenylene scaffold. This was achieved employing a Michael addition of the thiol containing peptides to maleimide functions on the dendrimer periphery. An important prerequisite for the synthesis of polyphenylene dendrimers decorated with two different peptide units is the creation of a core comprising two types of anchor groups, allowing the independent coupling of different peptide sequences.

\section{EXPERIMENTAL SECTION}

General Information. Unless stated otherwise, all solvents and reagents are commercially available and were used as received. ${ }^{1} \mathrm{H}$ NMR spectra were recorded on Bruker AMX 250, Bruker AMX 300, Bruker Advance DRX 500 or 700 spectrometers. Spectra were referenced to the residual proton signal of the deuterated solvent. Molecular weights were determined with matrix-assisted laser desorption/ionization time-of-flight (MALDI-TOF) or field desorption (FD) mass spectrometry; MALDI-TOF mass spectra were recorded on a Bruker MALDI-TOF mass spectrometer, and FD mass spectra were recorded on VG Instruments ZAB 2 Se-FPD. Peptide syntheses were carried out on an Applied Biosystems peptide synthesizer model 433A. Ultraviolet-visible (UV-vis) spectra and fluorescence spectra were recorded using a Perkin-Elmer Lambda 9 and SPEX Fluorolog 2 spectrophotometer, respectively. Circular dichroism (CD) spectra were measured on a Jasco J-715 spectropolarimeter. Gel permeation chromatography (GPC) was performed at 60 ${ }^{\circ} \mathrm{C}$ with a setup consisting of a Waters 510 pump and a series of three styrene-divinylbenzene columns $(300 \times 8$ $\mathrm{mm}$ ) with pore sizes of $500,10^{5}$, and $10^{6} \AA$ (Polymer Standard Services, Mainz, Germany). The elemental analyses were carried out by the Microanalytical Laboratory of the University of Mainz (Germany).

The amino acids were free acids, and coupling was facilitated by the use of $O$-benzotriazole- $N, N, N^{\prime}, N^{\prime}$-tertramethyluronium hexafluorophosphate (HBTU) and $N$ hydroxybenzotriazole (HOBt). Purity was checked using analytical reverse-phase high performance liquid chromatography (RP-HPLC, AKTA-Purifier, AmershamPharmacia Biotech). The column used was a reversephase C8 column (Sephasil peptide C8, volume $4.155 \mathrm{~mL}$, $5 \mu \mathrm{m}$ particle size). The synthesis of first- and secondgeneration dendrimers with maleimide functionalities $\mathbf{1 3 a}-\mathbf{d}$ and conjugates $\mathbf{3 8 a}-\mathbf{d}$ have been described previously (10). Compounds 1 (11), 2 (12), 3 (13), 4 (14), and 16 (15) were synthesized according published procedures.

Procedures. Compound 7a. 3-(4-Aminophenyl)-2,4,5triphenylcyclopenta-2,4-dienone (6a) $(1.4 \mathrm{~g}, 3.55 \mathrm{mmol})$, $N$-BOC- $\gamma$-aminobutyric acid (2.4 g, $17.71 \mathrm{mmol}), \mathrm{N}$-(3dimethylaminopropyl)- $N$ '-ethylcarbodiimide hydrochloride (EDC) (2.4 g, $17.71 \mathrm{mmol})$, and DMAP (1.3 g, 10.6 $\mathrm{mmol}$ ) were dissolved in $50 \mathrm{~mL}$ of DMF and stirred for $48 \mathrm{~h}$ under an argon atmosphere. Thereafter, DCM (50 $\mathrm{mL}$ ) was added, and the reaction mixture was washed twice with water. The organic phase was isolated, dried over magnesium sulfate, and filtrated, and the solvent was evaporated. The dried residue was purified by column chromatography with DCM/acetone (10:1) eluent to give $\mathbf{7 a}$ as brown powder. Yield: $2.07 \mathrm{~g}(82.1 \%)$. FD $\left(\mathrm{M}^{+}\right): m / z=585.5 ;{ }^{1} \mathrm{H} \mathrm{NMR}\left(250 \mathrm{MHz}, \mathrm{CD}_{2} \mathrm{Cl}_{2}\right) \delta_{\mathrm{H}} 7.43$ (d, $2 \mathrm{H}, J=8.48 \mathrm{~Hz}), 7.32-7.18$ (m, 13H, arom.), 6.98 (d, $2 \mathrm{H}, J=7.85 \mathrm{~Hz}), 6.87(\mathrm{~d}, 2 \mathrm{H}, J=7.89 \mathrm{~Hz}), 3.21(\mathrm{q}, 2 \mathrm{H}$, $J=6.28 \mathrm{~Hz}), 2.23(\mathrm{t}, 2 \mathrm{H}, J=6.28 \mathrm{~Hz}), 1.81(\mathrm{t}, 2 \mathrm{H}, J=$ $6.28 \mathrm{~Hz}), 1.44(\mathrm{~s}, 9 \mathrm{H}) ;{ }^{13} \mathrm{C}$ NMR $\left(75 \mathrm{MHz}, \mathrm{DMSO}-d_{6}\right) \delta_{\mathrm{C}}$ $199.6,171.1,171.0,155.5,154.5,139.6,139.5,132.7$, $130.7,130.5,129.7,129.6,128.8,128.5,128.0,127.9$, $127.5,127.4,126.8,124.9,124.1,118.1,117.9,77.4,33.7$, 28.2, 25.3, $11.0 ; \mathrm{mp} 106{ }^{\circ} \mathrm{C}$.

Compound 7b. 3,4-Bis-(4-aminophenyl)-2,5-diphenylcyclopenta-2,4-dienone (6b) (1.1 g, $2.69 \mathrm{mmol}), N$-BOC$\gamma$-aminobutyric acid $(5.46 \mathrm{~g}, 26.9 \mathrm{mmol}), \mathrm{EDC}(5.16 \mathrm{~g}$, $26.9 \mathrm{mmol}$ ), and DMAP (1.97 g, $16.1 \mathrm{mmol}$ ) were dissolved in $50 \mathrm{~mL}$ of DMF and stirred for $48 \mathrm{~h}$ under an argon atmosphere. DCM $(50 \mathrm{~mL})$ was added, the reaction mixture was washed twice with water, and the organic phase isolated, dried over magnesium sulfate, and filtrated, and the solvent was evaporated. The dried residue was purified by column chromatography with DCM/ acetone $(2: 1)$ as eluent to give $\mathbf{7 b}$ as brown powder. Yield: $1.54 \mathrm{~g}(73 \%)$. FD $\left(\mathrm{M}^{+}\right): \mathrm{m} / z=782.6 ;{ }^{1} \mathrm{H}$ NMR $(250$ $\left.\mathrm{MHz}, \mathrm{DMSO}-d_{6}\right) \delta_{\mathrm{H}} 7.43(\mathrm{~d}, 2 \mathrm{H}, J=8.85 \mathrm{~Hz}), 7.30-7.13$ (m, 10H, arom.), $6.83(\mathrm{~d}, 2 \mathrm{H}, J=8.53 \mathrm{~Hz}), 2.92(\mathrm{q}, 4 \mathrm{H}$, $J=6.63 \mathrm{~Hz}), 2.26(\mathrm{t}, 4 \mathrm{H}, J=7.27 \mathrm{~Hz}), 1.65(\mathrm{p}, 4 \mathrm{H}, J=$ $7.27 \mathrm{~Hz}), 1.35(\mathrm{~s}, 18 \mathrm{H}) ;{ }^{13} \mathrm{C}$ NMR $\left(75 \mathrm{MHz}, \mathrm{DMSO}-d_{6}\right) \delta_{\mathrm{C}}$ $199.8,171.2$, 155.8, 154.5, 139.8, 139.7,139.0, 130.0, $129.9,128.2,127.6,127.1,124.5,118.2,77.6,34.0,28.5$, $25.5,11.2$.

Compound 8a. This compound was prepared analogously to 7a using $\mathbf{6 a}(1.4 \mathrm{~g}, 3.55 \mathrm{mmol}), N-Z-\gamma$-aminobutyric acid (3.37 g, $14.2 \mathrm{mmol}), \mathrm{EDC}(2.72 \mathrm{~g}, 14.2 \mathrm{mmol})$, and DMAP (1.04 g, $8.5 \mathrm{mmol})$ in $50 \mathrm{~mL}$ of DMF. The crude product was purified by column chromatography with DCM/acetone (10:1) as eluent to give 8a as brown powder. Yield: $1.8 \mathrm{~g}(82 \%)$. FD $\left(\mathrm{M}^{+}\right): \mathrm{m} / z=619.4 ;{ }^{1} \mathrm{H}$ NMR $\left(250 \mathrm{MHz}, \mathrm{DMSO}-d_{6}\right) \delta_{\mathrm{H}} 7.44-7.12(\mathrm{~m}, 20 \mathrm{H}$, arom.), $6.96(\mathrm{~d}, 2 \mathrm{H}, J=7.9 \mathrm{~Hz}), 6.83(\mathrm{~d}, 2 \mathrm{H}, J=8.85$ $\mathrm{Hz}), 4.99$ (s, 2H), 3.01 (q, 2H, $J=6.64 \mathrm{~Hz}), 2.89$ (t, $2 \mathrm{H}$, $J=7.26 \mathrm{~Hz}), 1.75(\mathrm{p}, 2 \mathrm{H}, J=6.63 \mathrm{~Hz}) ;{ }^{13} \mathrm{C} \mathrm{NMR}(75$ 
$\left.\mathrm{MHz}, \mathrm{DMSO}-d_{6}\right) \delta_{\mathrm{C}} 199.7,171.1,156.1,154.6,154.5$, $139.6,139.5,137.2,135.8,132.8,130.7,130.5,129.8$, $129.7,128.9$, 128.6, 128.4, 128.3, 128.0, 127.9, 127.7, $127.5,127.4,126.8,125.0,124.1,118.0,106.1,65.1,46.1$, $33.6,25.3 ; \operatorname{mp} 80^{\circ} \mathrm{C}$

Compound 8b. This compound was prepared analogously to $7 \mathbf{b}$ using $\mathbf{6 b}(1.1 \mathrm{~g}, 2.69 \mathrm{mmol}), N$-Z $-\gamma$-aminobutyric acid (6.39 g, $26.9 \mathrm{mmol})$, EDC (7.92 g, $26.9 \mathrm{mmol})$, and DMAP (1.97 g, $16.1 \mathrm{mmol})$ in $50 \mathrm{~mL}$ of DMF. The crude product was purified by column chromatography with DCM/acetone (2:1) as eluent to give $\mathbf{8 b}$ as brown powder. Yield: $1.75 \mathrm{~g}(76.2 \%)$. FD $\left(\mathrm{M}^{+}\right): \mathrm{m} / z=853 ;{ }^{1} \mathrm{H}$ NMR $\left(250 \mathrm{MHz}, \mathrm{DMSO}-d_{6}\right) \delta_{\mathrm{H}} 7.44(\mathrm{~d}, 4 \mathrm{H}, J=8.53 \mathrm{~Hz})$, $7.33-7.13$ (m, 24H, arom.), $6.84(\mathrm{~d}, 4 \mathrm{H}, J=8.53 \mathrm{~Hz}$ ), $4.99(\mathrm{~s}, 4 \mathrm{H}), 3.02(\mathrm{q}, 4 \mathrm{H}, J=6.32 \mathrm{~Hz}), 2.29(\mathrm{t}, 4 \mathrm{H}, J=$ $7.27 \mathrm{~Hz}), 1.65(\mathrm{p}, 4 \mathrm{H}, J=5.95 \mathrm{~Hz}) ;{ }^{13} \mathrm{C} \mathrm{NMR}(75 \mathrm{MHz}$, DMSO- $\left.d_{6}\right) \delta_{\mathrm{C}} 199.8,171.3,171.2,156.3,154.5,139.8$, $139.7,137.4,131.0,130.0,128.5,128.5,127.9,127.6$, 127.1, 124.6, 118.3, 65.4, 46.4, 33.8, 25.3; mp $248^{\circ} \mathrm{C}$.

Compound 9. This compound was prepared analogously to 7a using $6 \mathbf{a}(0.7 \mathrm{~g}, 1.8 \mathrm{mmol})$, hexanedioic acid monomethyl ester (1.44 g, $9 \mathrm{mmol})$, EDC (1.72 g, $9 \mathrm{mmol})$, and DMAP $(0.66 \mathrm{mg}, 5.4 \mathrm{mmol})$ in $50 \mathrm{~mL}$ of DMF. The crude product was purified by column chromatography with DCM/acetone (50:1) as eluent to give 9 as brown powder. Yield: $0.830 \mathrm{~g}(85 \%)$. FD $\left(\mathrm{M}^{+}\right): \mathrm{m} / z=542 ;{ }^{1} \mathrm{H}$ NMR $\left(250 \mathrm{MHz}, \mathrm{CD}_{2} \mathrm{Cl}_{2}\right) \delta_{\mathrm{H}} 7.51-7.16$ (m, 15H, arom.), $6.96(\mathrm{~d}, 2 \mathrm{H}, J=6.91 \mathrm{~Hz}), 6.85(\mathrm{~d}, 2 \mathrm{H}, J=8.5 \mathrm{~Hz}), 3.63$ (s, 3H), 2.49-2.25 (m, 4H), 1.80-1.58 (m, 4H); ${ }^{13} \mathrm{C}$ NMR $\left(75 \mathrm{MHz}, \mathrm{CD}_{2} \mathrm{Cl}_{2}\right) \delta_{\mathrm{C}} 199.9,179.0,130.8,130.7,129.8$, 129.0, 128.6, 128.5, 128.4, 127.9, 119.1, 51.9, 34.1, 24.8, 24.7 .

Compound 14a. Compounds $3(0.1 \mathrm{~g}, 0.085 \mathrm{mmol})$ and $7 \mathbf{a}(0.597 \mathrm{~g}, 1.02 \mathrm{mmol})$ were dissolved in $31.5 \mathrm{~mL}$ of a $o$-xylene/toluene/DMSO mixture (5/5/2). The resulting solution was heated at $150{ }^{\circ} \mathrm{C}$ for $36 \mathrm{~h}$ under an argon atmosphere. The reaction mixture was precipitated in petroleum ether, and the crude product was purified by chromatography (silica gel, $\mathrm{CH}_{2} \mathrm{Cl}_{2}$ /acetone 2:1) to give 14a. Yield: $202 \mathrm{mg}$ (70\%). MALDI-TOF MS ((M + $\left.\mathrm{Na})^{+}\right): m / z=3426 ;{ }^{1} \mathrm{H}$ NMR $\left(250 \mathrm{MHz}, d^{6}-\mathrm{DMSO}\right) \delta_{\mathrm{H}}$ $9.65,9.59(2 \mathrm{H}, \mathrm{NH}), 7.91$ (s, 4H, arom.), $7.42(\mathrm{~s}, 4 \mathrm{H}$, arom.), 7.33-6.66 (m, 98H, arom.), 2.89 (q, 8H, ${ }^{3} J=6.63$ $\mathrm{Hz}$ ), 2.69 (sept, $\left.4 \mathrm{H},{ }^{3} J=6.32 \mathrm{~Hz}\right), 2.17\left(\mathrm{q}, 8 \mathrm{H},{ }^{3} J=7.27\right.$ $\mathrm{Hz}), 1.59\left(\mathrm{p}, 8 \mathrm{H},{ }^{3} J=7.27 \mathrm{~Hz}\right), 1.34(\mathrm{~s}, 36 \mathrm{H}), 1.02(\mathrm{~d}$, $\left.24 \mathrm{H},{ }^{3} J=5.68 \mathrm{~Hz}\right),{ }^{13} \mathrm{C} \mathrm{NMR}\left(125 \mathrm{MHz}, \mathrm{DMSO}-d_{6}\right) \delta_{\mathrm{C}}$ $170.4,170.3,170.3,162.4,155.3,154.9,153.2,145.5$, $141.3,141.2,141.1,141.0,140.4,140.2,139.8,139.5$, $139.4,139.3,139.2,139.1,139.0,138.9,138.8,138.7$, $137.5,136.7,136.6,136.4,136.3,134.3,134.0,132.3$, $131.0,131.0,131.0,130.9,130.8,130.5,130.2,130.1$, $129.3,129.0,127.3,127.3,126.6,126.5,126.3,126.0$, $125.4,125.3,125.2,123.4,122.5,119.9,119.7,119.3$, 118.5, 117.3, 117.0, 77.3, 33.6, 28.1, 25.2, 23.3.

Compound 14b. Compounds $3(0.1 \mathrm{~g}, 0.085 \mathrm{mmol})$ and $7 \mathbf{b}(1.07 \mathrm{~g}, 2.04 \mathrm{mmol})$ were dissolved in $42 \mathrm{~mL}$ of a $o$-xylene/toluene/DMSO mixture $(5 / 5 / 2)$. The resulting solution was heated for $36 \mathrm{~h}$ at $150{ }^{\circ} \mathrm{C}$ under an argon atmosphere. The reaction mixture was precipitated in petroleum ether, and the crude product was purified by chromatography (silica gel, $\mathrm{CH}_{2} \mathrm{Cl}_{2}$ /acetone $1: 1$ ) to give 14b. Yield: $315 \mathrm{mg}$ (88\%). MALDI-TOF MS ((M + $\left.\mathrm{Na})^{+}\right): m / z=4225 ;{ }^{1} \mathrm{H}$ NMR $\left(250 \mathrm{MHz}, \mathrm{DMSO}-d_{6}\right) \delta_{\mathrm{H}}$ $9.66,9.61(2 \mathrm{~s}, \mathrm{NH}), 7.90(\mathrm{~s}, 4 \mathrm{H}$, arom. $), 7.43-6.68(\mathrm{~m}$, 94H, arom.), 2.88 (q, $16 \mathrm{H},{ }^{3} J=6.32 \mathrm{~Hz}$ ), 2.69 (sept, $4 \mathrm{H}$, $\left.{ }^{3} J=6.31 \mathrm{~Hz}\right), 2.17\left(\mathrm{q}, 16 \mathrm{H},{ }^{3} J=7.27 \mathrm{~Hz}\right), 1.60(\mathrm{p}, 16 \mathrm{H}$, $\left.{ }^{3} J=7.27 \mathrm{~Hz}\right), 1.34(\mathrm{~s}, 72 \mathrm{H}), 1.02\left(\mathrm{~d}, 24 \mathrm{H},{ }^{3} J=5.68 \mathrm{~Hz}\right)$, ${ }^{13} \mathrm{C}$ NMR $\left(125 \mathrm{MHz}, \mathrm{DMSO}-d_{6}\right) \delta_{\mathrm{C}} 170.4,170.3,170.2$, $162.4,155.3,154.9,153.2,145.5,141.2,140.4,139.4$,
$139.1,139.0,137.6,136.7,136.6,136.4,136.3,134.4$, $134.1,132.3,131.0,131.0,130.9,130.5,130.1,129.3$, $129.0,127.3,126.6,126.0,125.9,125.4,123.4,122.5$, $119.9,119.7,119.3,118.5,117.4,117.1,117.0,77.3,33.6$, $28.2,25.2,23.4$.

Compound 15a. Compound 14a (0.2 g, $0.06 \mathrm{mmol})$ was dissolved in $4 \mathrm{~mL}$ of $50 \%$ trifluoroacetic acid in dichloromethane and stirred at room temperature for 2 h. The reaction mixture was added to saturated $\mathrm{NaHCO}_{3}$ solution, and the precipitated product 15a was filtrated and dried under reduced pressure. Yield: $172 \mathrm{mg}(98 \%)$. MALDI-TOF MS $\left((\mathrm{M}+\mathrm{Na})^{+}\right): \mathrm{m} / z=3023 ;{ }^{1} \mathrm{H}$ NMR $(500$ $\left.\mathrm{MHz}, \mathrm{DMSO}-d_{6}\right) \delta_{\mathrm{H}} 7.93(\mathrm{~s}, 4 \mathrm{H}$, arom. $), 7.45-7.42(\mathrm{~m}$, $6 \mathrm{H}$, arom.), 7.31 (d, $4 \mathrm{H},{ }^{3} J=7.62 \mathrm{~Hz}$, arom.), 7.17-6.68 (m, 92H, arom.), 2.70 (sept, $\left.4 \mathrm{H},{ }^{3} J=6.41 \mathrm{~Hz}\right), 2.23$ (q, $\left.8 \mathrm{H},{ }^{3} J=7.63 \mathrm{~Hz}\right), 1.60\left(\mathrm{p}, 8 \mathrm{H},{ }^{3} J=6.72 \mathrm{~Hz}\right), 1.24(\mathrm{~s}$, $\mathrm{NH}), 1.04\left(\mathrm{~d}, 24 \mathrm{H},{ }^{3} J=6.41 \mathrm{~Hz}\right) ;{ }^{13} \mathrm{C} \mathrm{NMR}(75 \mathrm{MHz}$, DMSO- $\left.d_{6}\right) \delta_{\mathrm{C}} 177.2,171.0,162.7,155.0,153.3,145.5$, $141.8,141.2,141.1,140.5,140.4,139.9,139.7,139.6$, $139.5,139.4,139.1,138.9,138.8,137.6,136.9,134.3$, $134.0,133.5,132.5,131.2,131.0,130.6,129.5,127.7$, $127.0,126.7,126.4,125.8,123.8,122.6,120.1,119.5$, 119.4, 118.9, 117.3, 116.9, 53.0, 44.3, 33.9, 28.4, 23.7.

Compound 15b. Compound $14 \mathrm{~b}(0.2 \mathrm{~g}, 0.05 \mathrm{mmol})$ was dissolved in $4 \mathrm{~mL}$ of $50 \%$ trifluoroacetic acid in dichloromethane and stirred at room temperature for 2 $\mathrm{h}$. The reaction mixture was added to a saturated $\mathrm{NaHCO}_{3}$ solution, and the precipitated product $\mathbf{1 5 b}$ was filtrated and dried under reduced pressure. Yield: 158 $\mathrm{mg}(98 \%)$. MALDI-TOF MS $\left((\mathrm{M}+\mathrm{Na})^{+}\right): \mathrm{m} / z=3423$; ${ }^{1} \mathrm{H}$ NMR $\left(250 \mathrm{MHz}, \mathrm{DMSO}-d_{6}\right) \delta_{\mathrm{H}} 7.92$ (s, $4 \mathrm{H}$, arom.), 7.44 (t, ${ }^{3} J=7.62 \mathrm{~Hz}, 2 \mathrm{H}$, arom.), 7.39 (s, 4H, arom.), 7.31 (d, ${ }^{3} J=7.62 \mathrm{~Hz}, 4 \mathrm{H}$, arom.), $7.19-6.69$ (m, 88H, arom.), 2.70 (sept, $\left.{ }^{3} J=6.72 \mathrm{~Hz}, 16 \mathrm{H}\right), 2.23\left(\mathrm{q},{ }^{3} J=7.63 \mathrm{~Hz}, 8 \mathrm{H}\right), 1.60$ $\left(\mathrm{p},{ }^{3} \boldsymbol{J}=6.72 \mathrm{~Hz}, 8 \mathrm{H}\right), 1.24(\mathrm{~s}, \mathrm{NH}), 1.04\left(\mathrm{~d}, 24 \mathrm{H},{ }^{3} \boldsymbol{J}=\right.$ $6.41 \mathrm{~Hz}) ;{ }^{13} \mathrm{C}$ NMR $\left(75 \mathrm{MHz}, \mathrm{DMSO}-d_{6}\right) \delta_{\mathrm{C}} 170.8,166.8$, $162.7,155.0,153.3,145.5,141.4,141.2,140.5,140.0$, $139.6,139.1,137.6,136.9,136.8,136.6,134.5,134.2$, $132.4,131.3,130.8,130.6,129.5,129.3,127.7,126.9$, $126.4,125.7,125.6,123.8,122.6,120.1,119.9,119.3$, $118.9,117.3,117.0,114.0,66.9,33.6,31.3,28.4,23.8$.

Compound 17a. Compounds 16 (0.1 g, $0.036 \mathrm{mmol})$ and $8 \mathbf{a}(0.797 \mathrm{~g}, 1.29 \mathrm{mmol})$ were dissolved in $9 \mathrm{~mL}$ of a tetraethyleneglycol/diphenyl ether mixture (1:1). The resulting solution was heated for $24 \mathrm{~h}$ at $170{ }^{\circ} \mathrm{C}$ under an argon atmosphere. The reaction mixture was precipitated in methanol, and the crude product was purified by chromatography (silica gel, $\mathrm{CH}_{2} \mathrm{Cl}_{2}$ /acetone 1:4) to give 17a. Yield: $197 \mathrm{mg}(73 \%)$. MALDI-TOF MS ((M + $\left.\mathrm{Na})^{+}\right): m / z=7640 ;{ }^{1} \mathrm{H}$ NMR $\left(300 \mathrm{MHz}, \mathrm{DMSO}-d_{6}\right) \delta_{\mathrm{H}}$ 9.64, $9.58(2 \mathrm{~s}, \mathrm{NH}), 7.92(\mathrm{~s}, 4 \mathrm{H}$, arom.), 7.37-6.69 (m, $298 \mathrm{H}$, arom.), 4.98 (s, 16H), 2.97 (br, 16H), 2.72 (sept, $\left.4 \mathrm{H},{ }^{3} J=6.31 \mathrm{~Hz}\right), 2.20(\mathrm{br}, 16 \mathrm{H}), 1.63(\mathrm{br}, 16 \mathrm{H}), 1.02$ $(\mathrm{br}, 24 \mathrm{H}) ;{ }^{13} \mathrm{C}$ NMR $\left(75 \mathrm{MHz}, \mathrm{DMSO}-d_{6}\right) \delta_{\mathrm{C}} 170.5,156.3$, $156.1,141.4,141.2,141.0,140.4,139.8,139.3,139.0$, $138.4,137.6,137.1,136.7,136.6,134.6,134.2,131.5$, $131.1,131.0,129.5,128.3,127.8,126.8,126.4,117.3$, $116.8,60.2,60.1,33.7,28.8,25.3,23.9$.

Compound 17b. Compounds 16 (0.12 g, $0.043 \mathrm{mmol})$ and $8 \mathbf{b}(0.879 \mathrm{~g}, 1.03 \mathrm{mmol})$ were dissolved in $18 \mathrm{~mL}$ of a tetraethyleneglycol/diphenyl ether mixture (1:1). The resulting solution was heated at $170{ }^{\circ} \mathrm{C}$ for $24 \mathrm{~h}$ under an argon atmosphere. The reaction mixture was precipitated in methanol and the crude product purified by chromatography (silica gel, $\mathrm{CH}_{2} \mathrm{Cl}_{2}$ /acetone 1:4) to give 17b. Yield: $286 \mathrm{mg}$ (71\%). MALDI-TOF MS ((M + $\left.\mathrm{Na})^{+}\right): m / z=9434 ;{ }^{1} \mathrm{H}$ NMR $\left(300 \mathrm{MHz}\right.$, DMSO- $\left.d_{6}\right) \delta_{\mathrm{H}}$ 9.65, $9.59(2 \mathrm{~s}, \mathrm{NH}), 7.90(\mathrm{~s}, 4 \mathrm{H}$, arom.), 7.31-6.43 (m, $298 \mathrm{H}$, arom.), 4.98 (s, 32H), 2.98 (br, 32H), 2.71 (sept, 
$\left.4 \mathrm{H},{ }^{3} J=6.31 \mathrm{~Hz}\right), 2.20(\mathrm{br}, 32 \mathrm{H}), 1.64(\mathrm{br}, 32 \mathrm{H}), 1.02$ (br, $24 \mathrm{H}) ;{ }^{13} \mathrm{C}$ NMR $\left(75 \mathrm{MHz}, \mathrm{DMSO}-d_{6}\right) \delta_{\mathrm{C}} 170.6,156.1$, $156.0,141.5,141.3,140.9,140.3,139.7,139.4,139.0$, $138.4,137.5,137.2,136.8,136.5,134.6,134.2,131.6$, $131.2,130.9,129.5,128.3,127.7,126.7,126.3,117.3$, $116.9,60.2,60.1,33.6,28.9,25.2,23.8$.

Compound 18a. Compound 17a $(0.26 \mathrm{~g}, 0.035 \mathrm{mmol})$ was dissolved in $6 \mathrm{~mL}$ of trifluoroacetic acid and $210 \mu \mathrm{L}$ of $5.7 \mathrm{M} \mathrm{HBr}$ in acetic acid was added. The reaction mixture was stirred at room temperature for $2 \mathrm{~h}$ and added to a saturated $\mathrm{NaHCO}_{3}$ solution. The precipitate 18a was filtrated and dried under reduced pressure. Yield: $215 \mathrm{mg}(97 \%) .{ }^{1} \mathrm{H}$ NMR $\left(300 \mathrm{MHz}, \mathrm{DMSO}-d_{6}\right) \delta_{\mathrm{H}}$ 7.95 (s, 4H, arom.), 7.03-7.80 (m, 246H, arom.), 2.04 (br, $16 \mathrm{H}), 1.82$ (br, $16 \mathrm{H}), 1.43(\mathrm{br}, \mathrm{NH}), 1.01(\mathrm{br}, 24 \mathrm{H}) ;{ }^{13} \mathrm{C}$ NMR $\left(75 \mathrm{MHz}, \mathrm{DMSO}-d_{6}\right) \delta_{\mathrm{C}} 170.0,162.7,158.3,157.8$, $155.0,153.3,145.5,141.8,141.3,140.9,140.3,140.1$, $139.9,139.6,139.3,138.9,138.6,138.4,137.9,137.6$, $137.1,136.7,136.2,134.6,134.3,132.5,131.9,131.3$, $130.9,130.7,130.6,129.9,129.5,129.2$, 129.0, 128.7, $128.3,128.0,127.7,126.8,126.6,126.4,125.5,123.8$, $122.6,120.1,119.3,118.9,117.3,116.9,115.3,49.9,32.8$, 28.3, 23.7, 22.

Compound 18b. Compound 17b $(0.23 \mathrm{~g}, 0.025 \mathrm{mmol})$ was dissolved in $6 \mathrm{~mL}$ of trifluoroacetic acid and $280 \mu \mathrm{L}$ of $5.7 \mathrm{M} \mathrm{HBr}$ in acetic acid were added. The reaction mixture was stirred at room temperature for $2 \mathrm{~h}$ and added to a saturated $\mathrm{NaHCO}_{3}$ solution. The precipitate 18b was filtrated and dried under reduced pressure. Yield: $170 \mathrm{mg}(96 \%) .{ }^{1} \mathrm{H}$ NMR $\left(300 \mathrm{MHz}, \mathrm{DMSO}-d_{6}\right) \delta_{\mathrm{H}}$ 7.90 (s, 4H, arom.), 7.69-6.44 (m, 238H, arom.), 2.29 (br, $32 \mathrm{H}), 1.76$ (br, 32H), 1.40 (br, NH), 1.18 (br, $24 \mathrm{H}) ;{ }^{13} \mathrm{C}$ NMR $\left(75 \mathrm{MHz}, \mathrm{DMSO}-d_{6}\right) \delta_{\mathrm{C}} 170.7,170.1,162.7,161.0$, $158.8,158.4,157.9,157.5,153.3,145.5,141.3,140.9$, $140.3,139.7,139.4,139.0,138.4,138.0,137.6,136.6$, $136.1,134.8,134.4,134.2,132.5,131.3,130.6,129.5$, $128.6,12.5,128.2,128.1,128.0,127.9,127.7,126.8,126.4$, $124.2,123.2,122.6,120.2,119.7,119.3,118.9,118.7$, 117.4, 117.0, 115.3, 47.6, 33.9, 32.9, 28.9, 23.8, 22.9.

Compound 19. Compounds $2(0.100 \mathrm{~g}, 0.137 \mathrm{mmol})$ and $\mathbf{5 b}(0.408 \mathrm{~g}, 0.55 \mathrm{mmol})$ in $o$-xylene $(15 \mathrm{~mL})$ were heated at $160{ }^{\circ} \mathrm{C}$ for $24 \mathrm{~h}$ under an argon atmosphere. The reaction mixture was concentrated to one-fourth of the initial volume, and the product was purified by precipitation from a mixture of methanol and water (7: 1) to give a yellow powder. Yield: $0.250 \mathrm{~g}(84 \%)$. MALDITOF MS $\left(\mathrm{M}^{+}\right): \mathrm{m} / z=2159 ;{ }^{1} \mathrm{H}$ NMR $\left(250 \mathrm{MHz}, \mathrm{CD}_{2} \mathrm{Cl}_{2}\right)$ $\delta_{\mathrm{H}} 7.72-6.70$ (m, 78H, arom.), 6.61-6.49 (m, 8H, arom.), 6.34-6.21 (m, 8H, arom.), $1.18-1.05$ (m, 42H); ${ }^{13} \mathrm{C} \mathrm{NMR}$ $\left(75 \mathrm{MHz}, \mathrm{CD}_{2} \mathrm{Cl}_{2}\right) \delta_{\mathrm{C}} 166.3,153.2,132.2,131.7,131.4$, $130.8,130.4,128.5,128.1,126.3,123.3,108.1,90.3,64.1$, $19.0,11.6$

Compound 20. To a solution of $\mathbf{1 9}(0.220 \mathrm{~g}, 0.102$ $\mathrm{mmol})$ in THF $(20 \mathrm{~mL})$ was added (tert-Bu $)_{4} \mathrm{NF} \cdot 3 \mathrm{H}_{2} \mathrm{O}$ $(0.130 \mathrm{~g}, 0.41 \mathrm{mmol})$. The reaction mixture was stirred for $5 \mathrm{~h}$ at room temperature. Water $(20 \mathrm{~mL})$ was added, and the product was extracted with DCM $(3 \times 15 \mathrm{~mL})$. The organic phase was isolated, dried over magnesium sulfate, filtrated, and concentrated to $5 \mathrm{~mL}$. The product was purified by precipitation from a mixture of methanol and water $(7: 1)$ to give a yellow powder. Yield: $0.160 \mathrm{~g}$ (85\%). MALDI-TOF MS ( $\left.\mathrm{M}^{+}\right): \mathrm{m} / z=1848 ;{ }^{1} \mathrm{H}$ NMR $(250$ $\left.\mathrm{MHz}, \mathrm{CD}_{2} \mathrm{Cl}_{2}\right) \delta_{\mathrm{H}} 7.74-6.70(\mathrm{~m}, 78 \mathrm{H}$, arom.), 6.62-6.50 (m, 8H, arom.), 6.35-6.22 (m, 8H, arom.), $3.12(\mathrm{~s}, 2 \mathrm{H})$; ${ }_{13} \mathrm{C} \mathrm{NMR}\left(75 \mathrm{MHz}, \mathrm{CD}_{2} \mathrm{Cl}_{2}\right) \delta_{\mathrm{C}} 166.3,153.2,132.2,131.8$, $131.4,130.8,130.4,128.5,128.1,126.3,123.3,85.7,79.4$, 64.1.

Compound 21. Compounds 20 (0.100 g, $0.054 \mathrm{mmol})$ and 9 (0.105 g, $0.204 \mathrm{mmol})$ were dissolved in $o$-xylene
(15 mL) and heated at $160{ }^{\circ} \mathrm{C}$ for $24 \mathrm{~h}$ under an argon atmosphere. The reaction mixture was concentrated to one-fourth of the initial volume, and the product was purified by precipitation from methanol to give a yellow powder. Yield: $0.130 \mathrm{~g}(84 \%)$. MALDI-TOF MS $\left(\mathrm{M}^{+}\right): \mathrm{m} / \mathrm{z}$ $=2875 ;{ }^{1} \mathrm{H}$ NMR $\left(250 \mathrm{MHz}, \mathrm{CD}_{2} \mathrm{Cl}_{2}\right) \delta_{\mathrm{H}} 7.74-6.21(\mathrm{~m}$, $134 \mathrm{H}$, arom.), $3.62(\mathrm{~s}, 6 \mathrm{H}), 2.32-2.18(\mathrm{~m}, 8 \mathrm{H}), 1.69-1.55$ $(\mathrm{m}, 8 \mathrm{H}) ;{ }^{13} \mathrm{C} \mathrm{NMR}\left(75 \mathrm{MHz}, \mathrm{CD}_{2} \mathrm{Cl}_{2}\right) \delta_{\mathrm{C}} 171.0,168.1$, $149.4,149.0,144.9,144.6,142.9,142.5,141.5,141.3$, $141.1,140.7,140.4,140.2,140.0,139.7,139.3,136.5$, $136.3,135.9,132.9,132.3,132.2,130.8,130.4,130.1$, $129.7,129.2,128.6,128.5,128.4,128.2,127.3,126.7$, $126.0,120.2,120.0,118.3,118.0,114.2,113.9,63.9,51.9$, 37.6, 34.1, 25.3, 24.9.

Compound 22. To a solution of $20(0.100 \mathrm{~g}, 0.035$ $\mathrm{mmol})$ in tetrahydrofuran $(2 \mathrm{~mL})$ was added an aqueous solution of $\mathrm{LiOH}(0.300 \mathrm{~mL}, 2.3 \mathrm{M})$, and the mixture was heated at $80^{\circ} \mathrm{C}$ for $12 \mathrm{~h}$ under an argon atmosphere. The reaction mixture was added to distilled water, and the product was extracted with DCM $(3 \times 20 \mathrm{~mL})$. The organic phases were collected, washed twice with water, and dried with $\mathrm{MgSO}_{4}$, and the solvent was evaporated to give 22 as yellow powder. Yield: $0.093 \mathrm{~g}(94 \%)$. MALDI-TOF MS: $m / z=2847 \mathrm{~g} / \mathrm{mol}\left(\mathrm{M}^{+}\right), 2869 \mathrm{~g} / \mathrm{mol}((\mathrm{M}$ $\left.+\mathrm{Na})^{+}\right) ;{ }^{1} \mathrm{H} \mathrm{NMR}\left(250 \mathrm{MHz}, \mathrm{CD}_{2} \mathrm{Cl}_{2}\right) \delta_{\mathrm{H}} 7.75-6.15(\mathrm{~m}$, $134 \mathrm{H}$, arom.), 2.16-1.55 (m, 16H); ${ }^{13} \mathrm{C} \mathrm{NMR}(75 \mathrm{MHz}$, $\left.\mathrm{CD}_{2} \mathrm{Cl}_{2}\right) \delta_{\mathrm{C}} 169.0,167.9,149.2,148.8,144.8,144.4,142.7$, $142.3,141.8,141.1,140.9,140.5,140.3,140.0,139.9$, $139.5,138.5,136.3,135.7,135.4,132.7,132.1,131.6$, $131.3,130.9,130.5,130.3,129.9,129.7,129.5,129.1$, $128.9,128.6,128.4,128.3,128.2,127.9,127.5,127.2$, $126.5,125.8,120.6,120.0,119.8,115.5,114.2,113.7,63.8$, 37.3, 30.1, 25.2.

Compound 23. Compound 22 (0.080 g, $0.032 \mathrm{mmol})$ was dissolved in THF $(0.5 \mathrm{~mL})$, and $2 \mathrm{~N} \mathrm{HCl}(1 \mathrm{~mL})$ added. After $10 \mathrm{~min}$, the mixture was cooled with ice and concentrated $\mathrm{HCl}(0.5 \mathrm{~mL})$ added. The mixture was stirred for $20 \mathrm{~min}$, and the solvents were evaporated under high vacuum. The final product was isolated as a bright brown powder, which was used in the next step without additional purification. Yield: $0.055 \mathrm{~g}(89 \%)$.

Compound 24. Compound 23 (0.065 g, $0.034 \mathrm{mmol})$ and 4-(2,5-dioxopyrrol-1-yl)butanoyl chloride $(0.085 \mathrm{~g}$, $0.41 \mathrm{mmol})$ were dissolved in a mixture of DMF (5 mL) and triethylamine $(0.5 \mathrm{~mL})$. The solution was stirred for $6 \mathrm{~h}$ at $\mathrm{RT}$ under an argon atmosphere. The product was purified by precipitation in methanol to give a dark yellow powder. Yield: $0.080 \mathrm{~g}(92 \%)$. MALDI-TOF MS $\left((\mathrm{M}+\mathrm{Na})^{+}\right) \mathrm{m} / z=2873 \mathrm{~g} / \mathrm{mol} ;{ }^{1} \mathrm{H} \mathrm{NMR}\left(250 \mathrm{MHz}, \mathrm{CD}_{2^{-}}\right.$ $\left.\mathrm{Cl}_{2}\right) \delta_{\mathrm{H}} 7.56-6.53(\mathrm{~m}, 102 \mathrm{H}$, arom.), 3.59-3.48 (m, 8), $2.45-2.12(\mathrm{~m}, 16 \mathrm{H}), 1.95-1.65(\mathrm{~m}, 16 \mathrm{H}) ;{ }^{13} \mathrm{C}$ NMR $(175$ $\left.\mathrm{MHz}, \mathrm{DMSO}-d_{6}\right) \delta_{\mathrm{C}} 174.1,170.9,169.9,162.2,143.7$, $141.2,140.2,140.0,139.5,139.0,136.7,136.4,134.3$, $132.9,131.2,130.0,129.5,128.5,128.4,127.6,126.8$, $126.6,126.2$, 125.4, 117.3, 116.9, 36.8, 35.7, 33.4, 30.7, 24.4, 24.1, 23.6.

Compounds 36a-d. The PDI dendrimers $15 \mathbf{a}, \mathbf{b}$ and 18a,b together with 5 equiv of peptide and 20 equiv of HOBT and HBTU were dissolved in a minimum quantity DMF and treated with 50 equiv of diisopropylethylamine (DIPEA). The reaction was stirred for 5 days at room temperature, and the product afterward was precipitated from diethyl ether. The resulting powder contained dendrimer-peptide-conjugate and peptide. Purification by dialysis proved impossible, and thus the mixture was dissolved in TFA and deprotected with $\mathrm{HBr}$ in acetic acid by the method described above. The crude product was precipitated from diethyl ether and subsequent ultrafil- 
Table 1. Synthesis and Properties of Peptide-Decorated PDI-Dendrimers ${ }^{a}$

\begin{tabular}{|c|c|c|c|c|c|c|c|c|c|c|}
\hline \multirow[b]{2}{*}{ sample } & \multirow[b]{2}{*}{ generation } & \multirow{2}{*}{$\begin{array}{c}Q_{\text {Initiator }} \\
{[\mathrm{mg}]}\end{array}$} & \multirow{2}{*}{$\begin{array}{c}Q_{\mathrm{NCA}} \\
{[\mathrm{mg}]}\end{array}$} & \multirow{2}{*}{$\begin{array}{l}\text { NMWL } \\
{[\mathrm{kDa}]}\end{array}$} & \multirow{2}{*}{$\begin{array}{l}\text { yield } \\
{[\mathrm{mg}]}\end{array}$} & \multirow{2}{*}{$\begin{array}{c}\text { yield } \\
{[\%]}\end{array}$} & \multirow{2}{*}{$\begin{array}{c}\text { theoretical } \\
m\end{array}$} & \multirow{2}{*}{$\begin{array}{l}\text { UV/ VIS } \\
m_{\text {Lys, UV }}\end{array}$} & \multicolumn{2}{|c|}{$\mathrm{GPC}_{\mathrm{DMF}}^{\mathrm{UV}}$} \\
\hline & & & & & & & & & $m_{\mathrm{Lys}, \mathrm{GPC}}$ & $M_{\mathrm{w}} / M_{\mathrm{n}}$ \\
\hline $30 \mathrm{a}$ & & 100 & 408 & 10 & 210 & 69.5 & 10 & 9 & 14 & 1.11 \\
\hline $30 \mathrm{~b}$ & $n=1$ & 22.5 & 459 & 30 & 195 & 72.5 & 50 & 50 & 54 & 1.16 \\
\hline $30 \mathrm{c}$ & $x=4$ & 14.3 & 587 & 50 & 286 & 84.3 & 100 & 85 & 84 & 1.15 \\
\hline $31 \mathrm{a}$ & & 66.4 & 479 & 10 & 265 & 88.3 & 10 & 9 & 12 & 1.09 \\
\hline $31 \mathrm{~b}$ & $n=1$ & 15.8 & 567 & 50 & 273 & 83.8 & 50 & 68 & 60 & 1.08 \\
\hline $31 \mathrm{c}$ & $x=8$ & 7.64 & 552 & 50 & 238 & 76.1 & 100 & 76 & 74 & 1.10 \\
\hline $32 \mathrm{a}$ & & 110.5 & 420 & 10 & 195 & 64.1 & 10 & 6 & 9 & 1.17 \\
\hline $32 \mathrm{~b}$ & $n=2$ & 27.7 & 526 & 30 & 175 & 55.7 & 50 & 28 & 22 & 1.42 \\
\hline $32 \mathrm{c}$ & & 15.1 & 572 & 50 & 120 & 34.8 & 100 & 41 & 37 & 1.27 \\
\hline $33 a$ & & 77.5 & 524 & 30 & 298 & 87.4 & 10 & 14 & 16 & 1.26 \\
\hline $33 \mathrm{~b}$ & $\begin{array}{l}n=2 \\
x-1\end{array}$ & 17.8 & 603 & 50 & 253 & 73.0 & 50 & 41 & 58 & 1.50 \\
\hline $33 \mathrm{c}$ & $x=16$ & 8.6 & 578 & 50 & 245 & 75.4 & 100 & 89 & 68 & 1.42 \\
\hline
\end{tabular}

${ }^{a}$ Notation: $x$, number of polypeptide chains per molecule; $m$, number of lysine residues per chain. The polydispersity $\left(M_{\mathrm{w}} / M_{\mathrm{n}}\right)$ of the peptide-dendrimer conjugates is calculated from the gel permeation chromatography data, obtained in DMF against polystyrene standards.

tration in water [nominal molecular weight limits (NMWLs) 10000 ] and freeze-drying yielded the products $\mathbf{3 6 a}-\mathbf{d}$.

36a. Yield: $159 \mathrm{mg}(61 \%) .{ }^{1} \mathrm{H}$ NMR $(700 \mathrm{MHz}, \mathrm{DMSO}-$ $\left.d_{6}\right) \delta_{\mathrm{H}} 7.91-6.70(\mathrm{~m}, 130 \mathrm{H}$, arom. $+\mathrm{NH}), 4.26-4.23(\mathrm{br}$, $20 \mathrm{H}), 3.96-3.10(\mathrm{~m}, \mathrm{NH}), 2.69$ (br, 40H), $2.22(\mathrm{br}, 8 \mathrm{H})$, $1.67-1.23(\mathrm{~m}, 120 \mathrm{H}), 1.05(\mathrm{br}, 24 \mathrm{H}), \mathrm{GPC}(\mathrm{UV}, \lambda=280$ $\mathrm{nm} ; \mathrm{PS}): M_{\mathrm{n}}=15700 \mathrm{~g} / \mathrm{mol}, M_{\mathrm{w}} / M_{\mathrm{n}}=1.1$.

36b. Yield: $169 \mathrm{mg}(48 \%) .{ }^{1} \mathrm{H}$ NMR (700 MHz, DMSO$\left.d_{6}\right) \delta_{\mathrm{H}} 7.90-6.71(\mathrm{~m}, 150 \mathrm{H}$, arom. $+\mathrm{NH}), 4.26-4.19(\mathrm{br}$, $\left.40 \mathrm{H}, \mathrm{H}_{\mathrm{c}}\right), 3.51-3.0(\mathrm{~m}, \mathrm{NH}), 2.71\left(\mathrm{br}, 80 \mathrm{H}, \mathrm{H}_{\mathrm{e}}\right), 2.24(\mathrm{br}$, $16 \mathrm{H}, \mathrm{Hb}), 1.67-1.24\left(\mathrm{~m}, 240 \mathrm{H}, \mathrm{H}_{\mathrm{d}}\right), 1.05\left(\mathrm{br}, 24 \mathrm{H}, \mathrm{H}_{\mathrm{a}}\right)$; GPC (UV, $\lambda=280 \mathrm{~nm}$; PS): $M_{\mathrm{n}}=20100 \mathrm{~g} / \mathrm{mol}, M_{\mathrm{w}} / M_{\mathrm{n}}=$ 1.1 .

36c. Yield: $129 \mathrm{mg}(52 \%) .{ }^{1} \mathrm{H}$ NMR (700 MHz, DMSO$\left.d_{6}\right) \delta_{\mathrm{H}} 7.95-6.49(\mathrm{~m}, 300 \mathrm{H}$, arom. $+\mathrm{NH}), 4.26-4.20(\mathrm{br}$, $40 \mathrm{H}, \mathrm{H}_{\mathrm{c}}$ ), 3-64-3.08 (m, NH), 2.75 (br, 80H, $\left.\mathrm{H}_{\mathrm{e}}\right), 2.24$ (br, $16 \mathrm{H}, \mathrm{Hb}), 1.68-1.24\left(\mathrm{~m}, 240 \mathrm{H}, \mathrm{H}_{\mathrm{d}}\right), 1.04(\mathrm{br}, 24 \mathrm{H}$, $\mathrm{H}_{\mathrm{a}}$ ); GPC (UV, $\lambda=280 \mathrm{~nm}$; PS): $M_{\mathrm{n}}=28600 \mathrm{~g} / \mathrm{mol}, M_{\mathrm{w}} /$ $M_{\mathrm{n}}=1.16$.

36d. Yield: $131 \mathrm{mg}(39 \%) .{ }^{1} \mathrm{H}$ NMR $(700 \mathrm{MHz}, \mathrm{DMSO}-$ $\left.d_{6}\right) \delta_{\mathrm{H}} 7.95-6.45(\mathrm{~m}, 340 \mathrm{H}$, arom. $+\mathrm{NH}), 4.30-4.15(\mathrm{br}$, $80 \mathrm{H}, \mathrm{H}_{\mathrm{c}}$ ), 3.35 (br, NH), $2.74\left(\mathrm{br}, 160 \mathrm{H}, \mathrm{H}_{\mathrm{e}}\right), 2.26(\mathrm{br}, 32 \mathrm{H}$, $\mathrm{Hb}), 1.68-1.04\left(\mathrm{~m}, 504 \mathrm{H}, \mathrm{H}_{\mathrm{d}+\mathrm{a}}\right)$; GPC (UV, $\lambda=280 \mathrm{~nm}$; PS): $M_{\mathrm{n}}=35800 \mathrm{~g} / \mathrm{mol}, M_{\mathrm{w}} / M_{\mathrm{n}}=1.12$.

Polymerization. An argon-filled and thoroughly dried Schlenk-tube fitted with a drying tube was charged with a filtered $(0.45 \mu \mathrm{m}$, PTFE) solution of the initiator (15ab and 18ab) in dry dimethylformamide. A solution NCA in dry dimethylformamide was added via a syringe driven filter, so that the final NCA concentration in the mixture reaches approximately $c=0.15-0.2 \mathrm{~g} / \mathrm{L}$. The mixture was stirred under the exclusion of light for 5 days at room temperature, and the product was precipitated by dropwise addition of a 15-fold (v/v) excess of water. The precipitate was filtered through a glass frit, washed extensively with $\mathrm{Et}_{2} \mathrm{O}$ to remove unreacted monomer, and dried under vacuum.

Deprotection of the Polymers. The polymer was dissolved in TFA, and a 4-fold excess (per lysine unit) of $\mathrm{HBr}$ in an $\mathrm{AcOH}$ solution $(5.7 \mathrm{~mol} / \mathrm{L})$ was added. After $1 \mathrm{~h}$ reaction time, the product was precipitated by the addition of $\mathrm{Et}_{2} \mathrm{O}$. The precipitate was then filtered through a glass frit, purified via ultrafiltration against Milli-Q-water until a constant conductivity was reached, and finally lyophilized. The membranes used had NMWLs of 10000 and 30000 (regenerated cellulose), and 50000 (polyethersulfone). All yields and molecular masses are listed in Table 1.

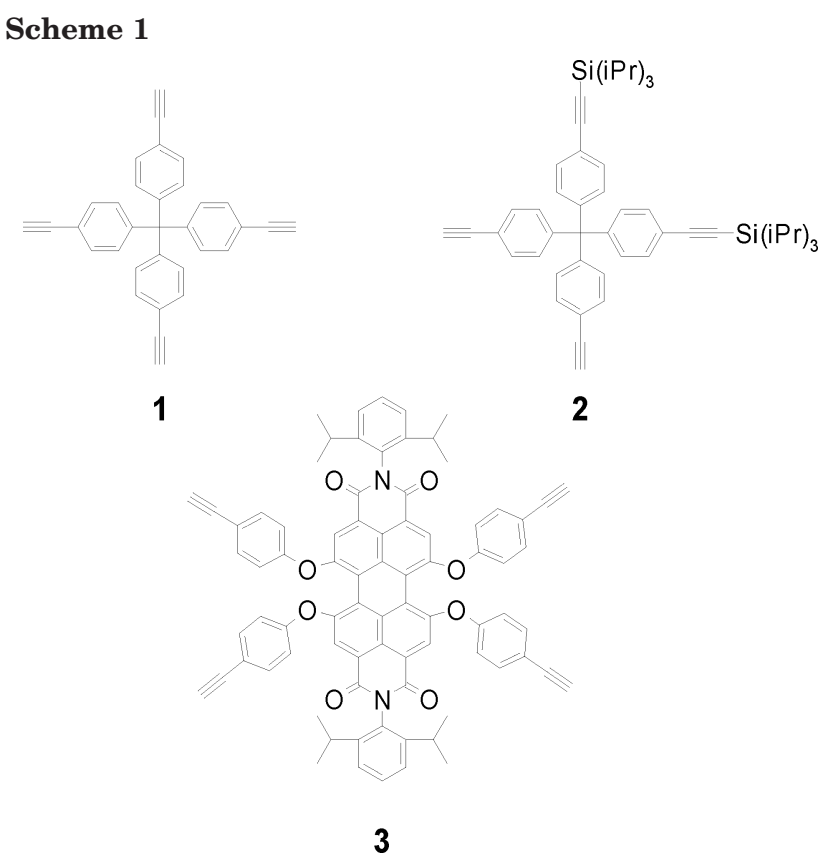

\section{RESULTS AND DISCUSSION}

The synthesis of polyphenylene dendrimers commences with a multi-ethynyl-substituted core molecule, namely, tetrakis-(4-ethynylphen-1-yl)-methane (1), its desymmetrized derivative $\mathbf{2}$, as well as the PDI core $\mathbf{3}$ (Scheme 1).

We emphasize that the geometry of the core determines the overall shape of the resulting cascade molecules (16). The growth of each dendritic layer is based upon an iterative process including a Diels-Alder reaction and subsequent deprotection of ethynyl functions (5). Scheme 2a introduces a functionalized cyclopentadienone 4 used as $\mathrm{A}_{2} \mathrm{~B}$-type branching agent (16). This molecule can act both as diene and after removal of the silyl protective groups from the ethynyl groups, as dienophile. In the [4+2]-cycloaddition between the cyclopentadienone and a free ethynyl group of the core molecule or the preceding dendrimer generation, a pentaphenylbenzene unit is formed under the extrusion of carbon monoxide (17-19).

3.1. Synthesis of Functionalized Cyclopentadienones. By using prefunctionalized cyclopentadienones $\mathbf{5 a b}, \mathbf{7 a b}, \mathbf{8 a b}, 9$ in the Diels-Alder reaction, polyphenylene dendrimers with chemical functions are obtained. These functions are either protected amino $(\mathbf{5 a b}, \mathbf{7 a b}$, $\mathbf{8 a b})$ or carboxyl (9) groups that can be readily converted into versatile anchor groups. The synthesis of the func- 
Scheme $2^{a}$

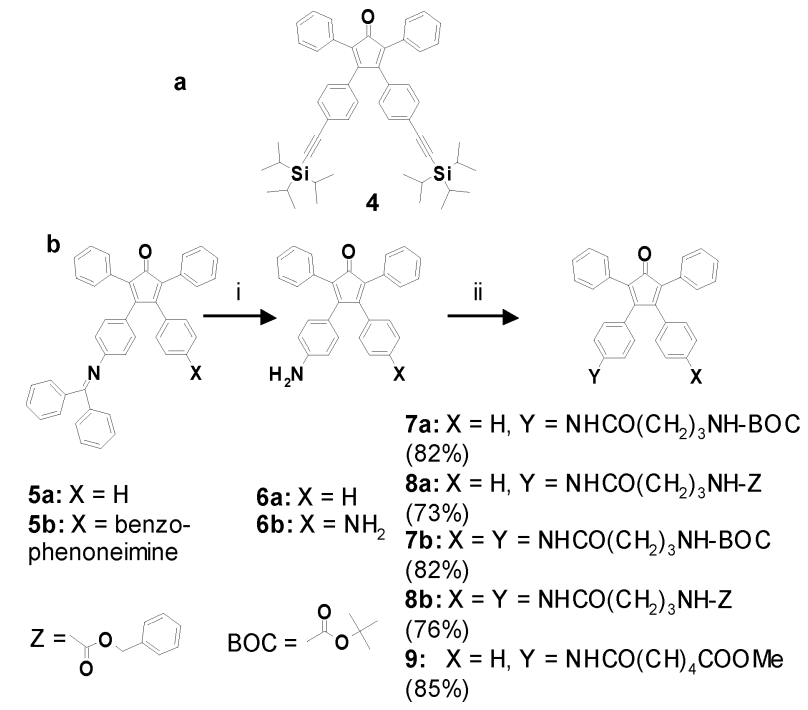

a (a) $\mathrm{A}_{2} \mathrm{~B}$-branching unit 3,4-bis-(4-triisopropylsilylethynylphenyl)-2,5-diphenyl-cyclopenta-2,4-dien-1-one 4; (b) synthesis of functionalized cyclopentadienones; i: $\mathrm{HCl}, \mathrm{RT}$, THF; ii: corresponding $N$-protected amino acid or hexanedioic acid monomethyl ester, EDC, DMAP, DMF, RT.

tionalized cyclopentadienones is depicted in Scheme $2 b$. The preparation of the imino- $(\mathbf{5 a}, \mathbf{b})$ and amino- $(\mathbf{6 a}, \mathbf{b})$ functionalized cyclopentadienones has been described previously (10).

$\gamma$-Aminobutyric acid-functionalized building blocks 7ab, 8ab were obtained by EDC-catalyzed coupling of

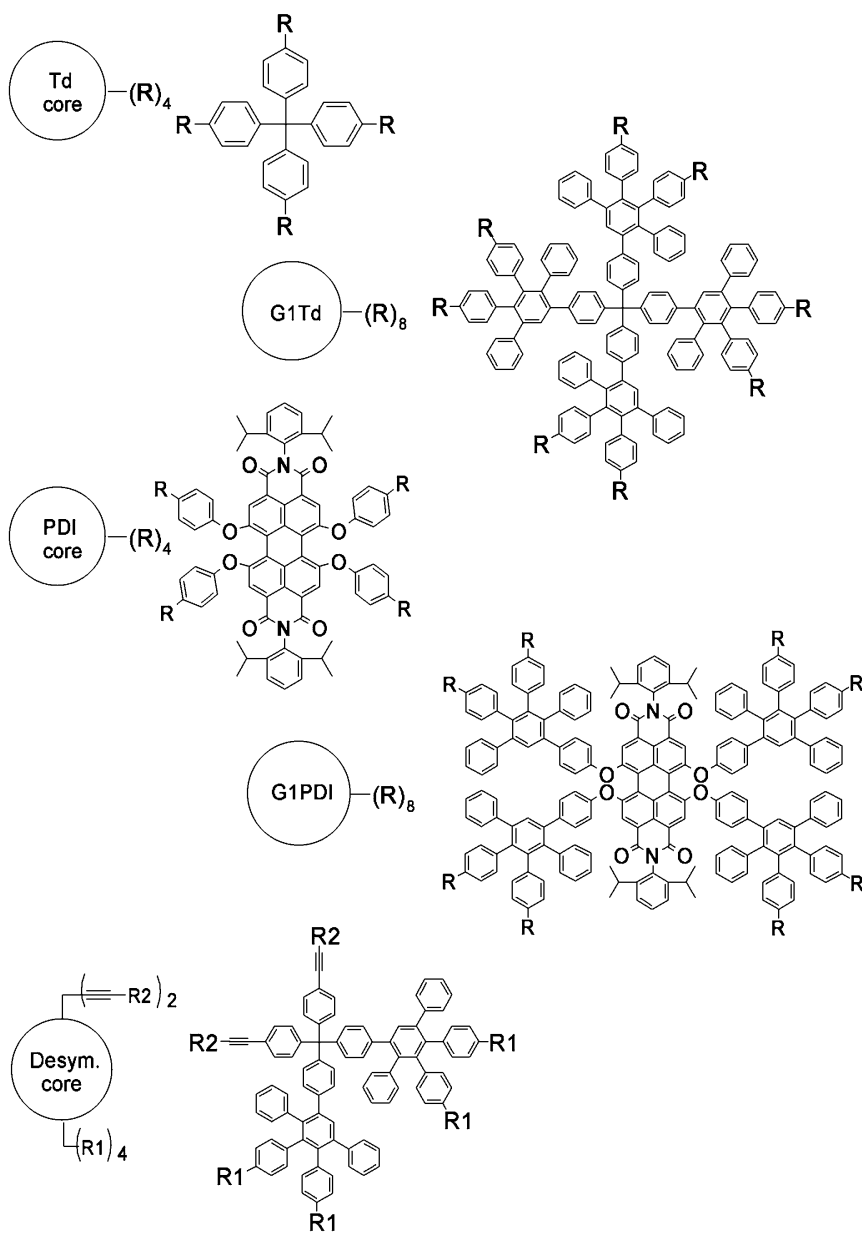

6a,b and the corresponding $N$-protected amino acid derivatives in DMF at room temperature. The products were purified by flash chromatography, and the structures were confirmed by ${ }^{1} \mathrm{H}$ NMR spectroscopy and FD mass spectrometry. Monocarboxy-functionalized cyclopentadienone 9 was synthesized analogously via a carbodiimide induced reaction of $\mathbf{6 a}$ with hexanedioic acid monomethyl ester.

3.2. Synthesis of Polyphenylene Dendrimers. For the sake of brevity, the terminology introduced in Figure 1 will be used for the central dendritic polyphenylene units.

Scheme 3 summarizes the synthesis of amino- and maleimide-functionalized dendrimers. We have previously described the preparation of maleimide-decorated polyphenylene dendrimers 13a-d (10). The Diels-Alder reaction of the functionalized cyclopentadienones $\mathbf{5 a b}$ with either the core $\mathbf{1}$ or the octaethynyl-functionalized dendrimer 10 yielded the benzophenoneimino dendrimers 11a-d. Subsequently, the complete cleavage of the protective groups was achieved with $\mathrm{HCl}$ in $\mathrm{THF}$ at room temperature, and the resulting amino dendrimers $12 \mathbf{a}-\mathbf{d}$ were reacted without additional purification with $4-(2,5-$ dioxo-pyrrol-1-yl)-butanoyl chloride to afford polyphenylene dendrimers with different numbers of maleimide anchor groups $\mathbf{1 3 a}-\mathbf{d}$.

The synthesis of first-generation BOC-protected aminofunctionalized PDI-dendrimers 14ab proceeded via the Diels-Alder cycloaddition of $\mathbf{7 a b}$ with the perylenediimide core 3. The subsequent removal of the BOC groups with trifluoroacetic acid in dichloromethane (20) afforded
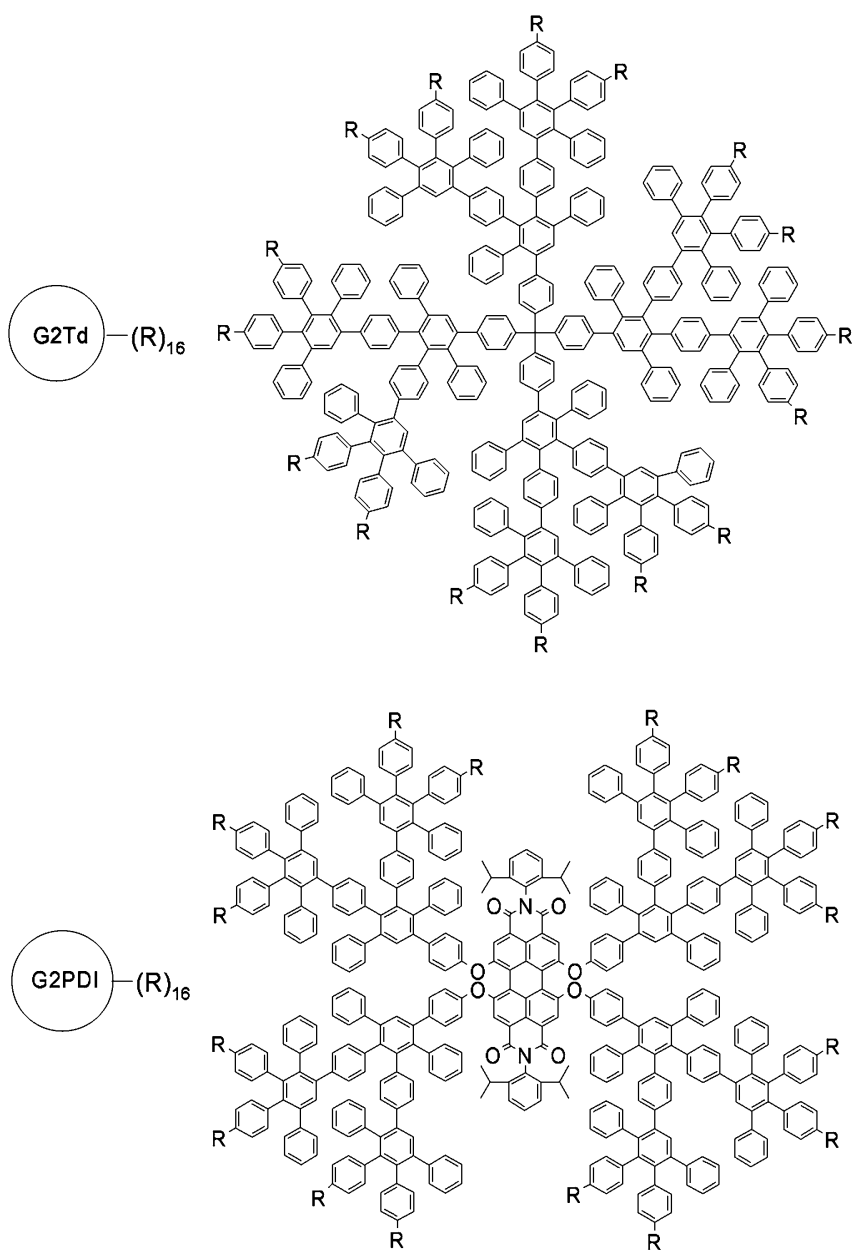

Figure 1. Symbols used to represent the polyphenylene scaffold of dendrimers in the schemes hereafter. The abbreviation in the circles shows the dendrimer generation and the central core unit. 
Scheme 3. Synthesis of Functionalized Dendrimers ${ }^{a}$

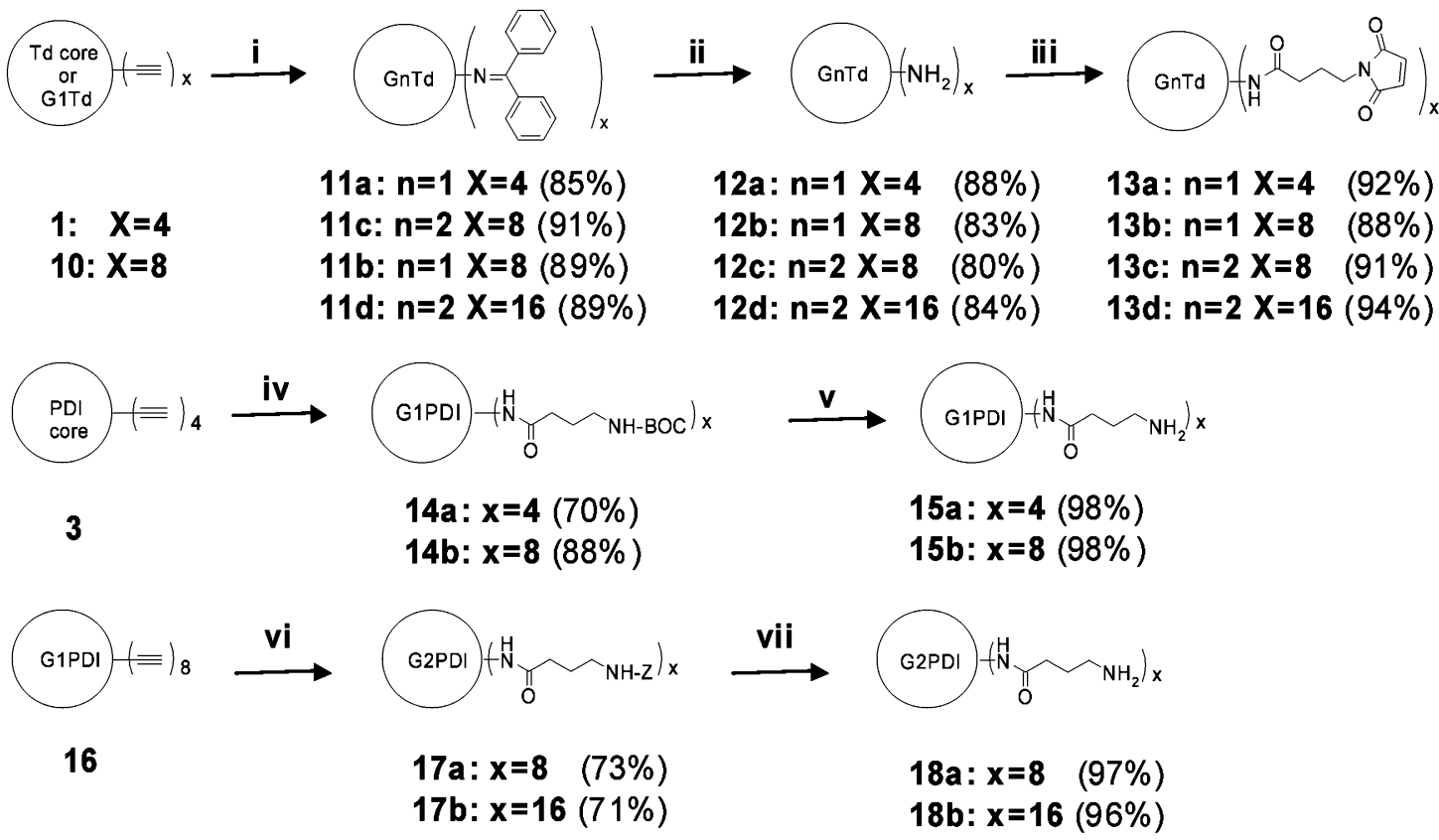

a (i) 5a/5b, $O$-xylene, $150{ }^{\circ} \mathrm{C}$; (ii) $\mathrm{HCl}$, THF; (iii) 4-(2,5-dioxo-2,5-dihydropyrrol-1-yl)butanoyl chloride, DMF, triethylamine; (iv) $\mathbf{7 a} / \mathbf{7 b}, O$-xylene, toluene, DMSO, $150^{\circ} \mathrm{C}$; (v) TFA, DCM, RT; (vi) 8a/8b, diphenyl ether, tetraethylene oxide, $170{ }^{\circ} \mathrm{C}$; (vii) $\mathrm{HBr}$, acetic acid. Notation: $\mathrm{n}$, generation of the dendrimer.

the amino compounds 15ab. Employing the reagents 8ab in a Diels-Alder reaction with $\mathbf{1 6}$ yielded the PDI dendrimers of the second generation $\mathbf{1 7 a b}$, which were readily converted to $\mathbf{1 8 a b}$ by treatment with $\mathrm{HBr}$ in glacial acetic acid (21). The amino groups of compounds 15ab and 18ab are more stable against oxidation and more accessible for the coupling of polypeptides than amino groups of the aniline derivatives $12 \mathbf{a}-\mathbf{d}$.

The synthesis of polyphenylene dendrimers possessing two different chemical functions is depicted in Scheme 4. First, cyclopentadienone $\mathbf{5 b}$ was reacted with the diethynyl core $\mathbf{2}$ to afford compound 19. After deprotection of the ethynyl groups, the subsequent Diels-Alder cycloaddition of $\mathbf{9}$ produced compound $\mathbf{2 0}$. Note that 20 has both (protected) amino and carboxyl anchor groups. The ester groups were converted to free carboxyl groups by $\mathrm{LiOH}$ in $\mathrm{THF}$ at $80{ }^{\circ} \mathrm{C}(22)$, while the deprotection of the amino groups was achieved by $\mathrm{HCl}$ in THF. Finally, the maleimide groups were introduced via reaction with 4-(2,5-dioxo-pyrrol-1-yl)-butanoyl chloride in $\mathrm{DMF}$ at room temperature to obtain the bifunctional compound $\mathbf{2 4}$.

3.3. Coupling of Polylysines to Polyphenylene Dendrimers. Scheme 5 gives an overview of the three synthetic routes toward peptide-decorated PPDs. Here we demonstrate the synthetic versatility of polyphenylene dendrimers as 3D carriers for the preparation of spatially defined bioactive conjugates. Three different strategies for peptide coupling were employed. We emphasize that the developed strategies are versatile and may be used for coupling of various oligopeptide fragments, according to the desired application.

3.3.1. Grafting of Protected Polypeptide from the PPD. The grafting of the polylysine from the surface of the polyphenylene dendrimers 15ab and 18ab was achieved by ring opening polymerization (Scheme 5a) of $\alpha$-amino acid $N$-carboxyanhydrides (NCA). The $\epsilon$-benzyloxycarbonyl-L-lysine $N$-carboxyanhydride (Lys $(\mathrm{Cbz})$ NCA) 25 was prepared following a literature procedure (23-25). 15a,b and $\mathbf{1 8 a}, \mathbf{b}$ can be regarded as macroinitiators to which the corresponding amount of Lys $(\mathrm{Cbz})$ -

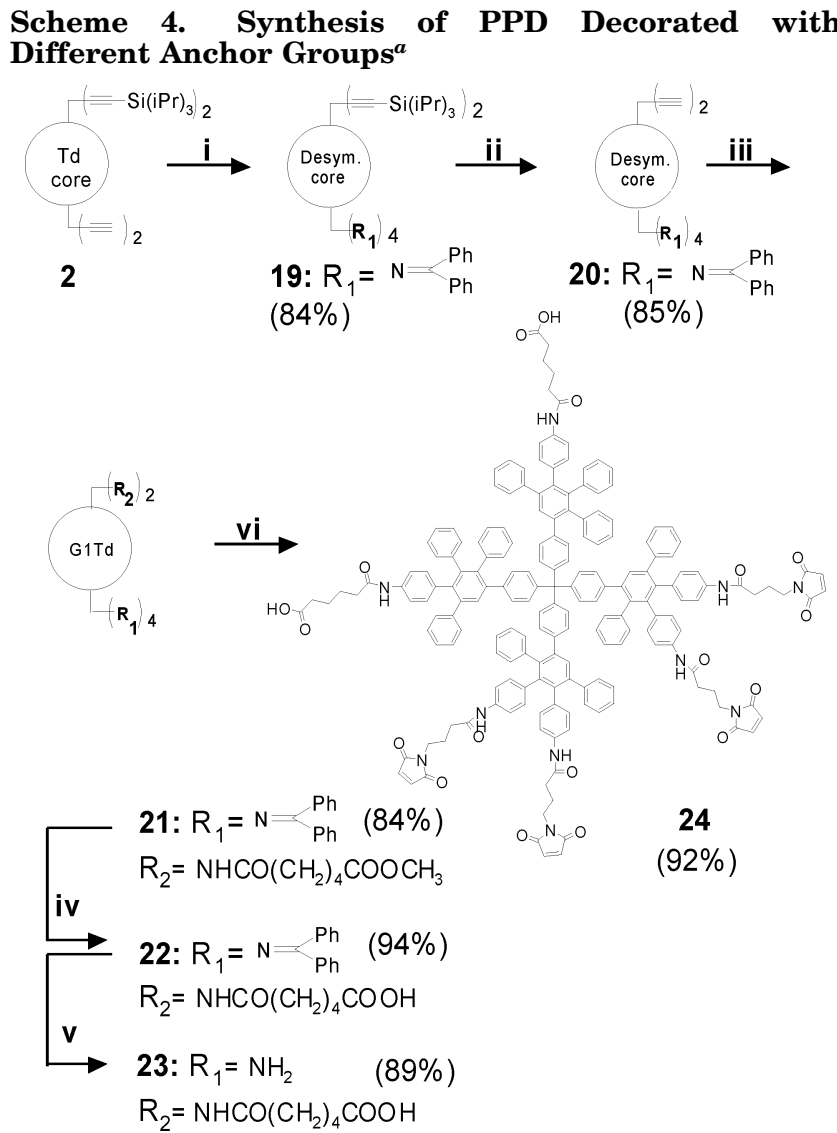

a (i) 5b, $O$-xylene, $145{ }^{\circ} \mathrm{C}$; (ii) $(\mathrm{t}-\mathrm{Bu})_{4} \mathrm{NF} \cdot 3 \mathrm{H}_{2} \mathrm{O}$, THF; (iii) $\mathbf{9}$, $O$-xylene, $145^{\circ} \mathrm{C}$; (iv) $\mathrm{LiOH}, \mathrm{THF}, 80^{\circ} \mathrm{C}$; (v) $2 \mathrm{~N} \mathrm{HCl}$, THF; (vi) 4-(2,5-dioxo-2,5-pyrrol-1-yl)-butanoyl chloride, DMF, triethylamine.

NCA was added, depending on the desired length of the peptide. The reaction proceeded in DMF at room temperature for 5 days.

The products $\mathbf{2 6 a}-\mathbf{c}, \mathbf{2 7 a}-\mathbf{c}, \mathbf{2 8 a}-\mathbf{c}, \mathbf{2 9 a}-\mathbf{c}$ were precipitated in water. Unreacted monomer was removed 
Scheme 5. Different Strategies for the Preparation of Peptide-dendrimer Conjugates ${ }^{a}$

a

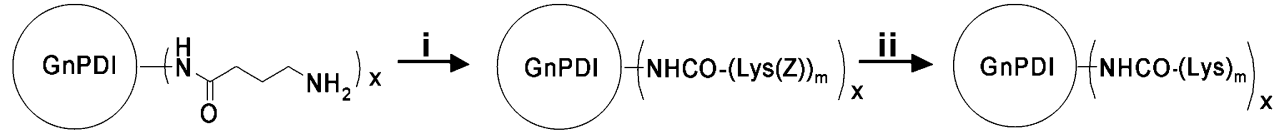

15a: $n=1, x=4 ; 15 b: n=1, x=8$

18a: $n=2, x=8 ; 18 b: n=2, x=16$

26-29 a-c

30-31a-c

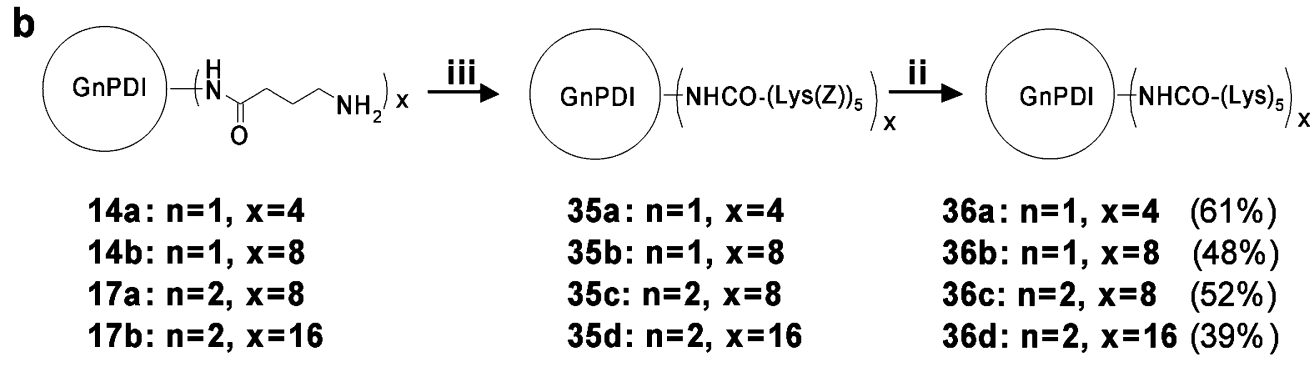

C
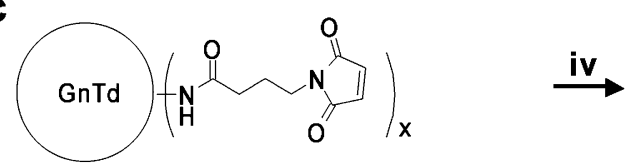

12a: $n=1, x=4$;

12b: $n=1, x=8$

12c: $n=2, x=8$;

12d: $n=2, x=16$

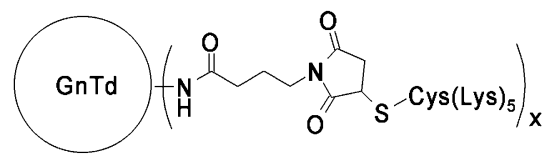
38a: $n=1, x=4(80 \%)$
38b: $n=1, x=8(65 \%)$
38c: $n=2, x=8(39 \%)$
38d: $n=2, x=16(45 \%)$

a (i) $\epsilon$-benzyloxycarbonyl-L-lysine $N$-carboxyanhydride (25), DMF; (ii) $\mathrm{HBr}$, acetic acid; (iii) HOOC-(Lys(Z)) 5 -NHZ (34), HBTU, HOBT, DIPEA, DMF; (iv) HOOC-Cys-(Lys) $)_{5}-\mathrm{NH}_{2}(\mathbf{3 7}), \mathrm{DMF}$. Notation: $\mathrm{n}$, generation of the dendrimer; m, number of lysine residues per chain.

by extensive washing with $\mathrm{Et}_{2} \mathrm{O}$. Finally, the deprotection of the side chains of the lysine residues yielded $\mathbf{3 0 a}-\mathbf{c}$, $\mathbf{3 1 a}-\mathbf{c}, \mathbf{3 2 a}-\mathbf{c}, \mathbf{3 3 a}-\mathbf{c}$ (Table 1). This reaction scheme permits control of the polypeptide length via the amount of the Lys $(\mathrm{Cbz})-\mathrm{NCA}$ added to the reaction. The dendrimers bear different numbers of polylysine chains of variable length (Table 1). These variable polypeptide lengths were used in the investigation of the secondary structure of the polylysine shell attached to a polyphenylene core (see section 2.4.). The Cbz-groups of the lysine residues were removed by $\mathrm{HBr}$ in glacial acetic acid, followed by precipitation in diethyl ether. To investigate the conjugates in salt-free solutions $\mathbf{3 0}-\mathbf{3 3 a}-\mathbf{c}$ were purified via ultrafiltration in milli-Q water.

3.3.2. Attachment of Protected Pentalysine. In this case, the $C$-terminus of a Cbz-protected pentalysine $\mathbf{3 4}$ is coupled to the amino groups on the surface of the PDI dendrimers (Scheme 5b). The peptide was obtained by solid-phase peptide synthesis using standard Fmoc protecting group chemistry (26). The activation of the $C$-terminal carboxyl group of the peptides was achieved using HOBT, HBTU, and DIPEA as activating agents (27).

The dendrimers $\mathbf{1 5 a}, \mathbf{b}$ or $\mathbf{1 8} \mathbf{a}, \mathbf{b}$, peptide $\mathbf{3 4}, \mathrm{HOBT}$, HBTU, and DIPEA were allowed to react for 5 days in dry DMF under an inert atmosphere at room temperature. After precipitation of the sample in diethyl ether, the crude product was found to contain the target compounds $\mathbf{3 5 a}-\mathbf{d}$ and the unreacted polypeptide. Since purification at this stage was not possible, the Z-groups were removed by the same protocol as mentioned in 3.3.1. Subsequent utrafiltration in water (NMWLs 10 000) and freeze-drying yielded the water soluble conjugates $\mathbf{3 6 a}-\mathbf{d}$. The structure and purity of these compounds were verified by means of ${ }^{1} \mathrm{H}$ NMR spectroscopy and gel permeation chromatography. The decoration of PDI dendrimers with preformed polypeptide sequences has the important advantage that no byproducts such as imperfect polypeptide chains occur. Note that the removal of such byproducts in the subsequent purification steps would be rather laborious (28).

3.3.3. Coupling of Unprotected Pentalysine. We also demonstrate a functionalization of the dendrimers with unprotected peptidesequences employing the Michael addition of the sulfhydryl group of terminal cysteine to the maleimide units on the dendrimer surface (Scheme 5c). Hexapeptide $\mathbf{3 7}$ is composed of a $C$-terminal cysteine and five lysine residues. The peptide was prepared via solid-phase peptide synthesis. The reaction of $\mathbf{1 3 a}-\mathbf{d}$ with $\mathbf{3 7}$ was performed in DMF at room temperature and the products $\mathbf{3 8 a}-\mathbf{d}$ were purified by dialysis and high performance liquid chromatography (HPLC). As for the method described in section 3.3.2, the absence of byproducts with wrong peptide sequences is a considerable advantage. Furthermore, in this case one circumvents the deprotection step(s), making this method especially convenient for coupling of polypeptide sequences sensitive to the deprotection procedures. It should be mentioned that the coupling via the $\mathrm{SH}$ group of the cysteine residue is not restricted to polypeptides.

3.4. Properties. 3.4.1. Circular Dichroism (CD). The intrinsic properties of proteins are achieved via secondary, tertiary, and quaternary structures. Therefore, a self-organization of the polypeptide shell attached 

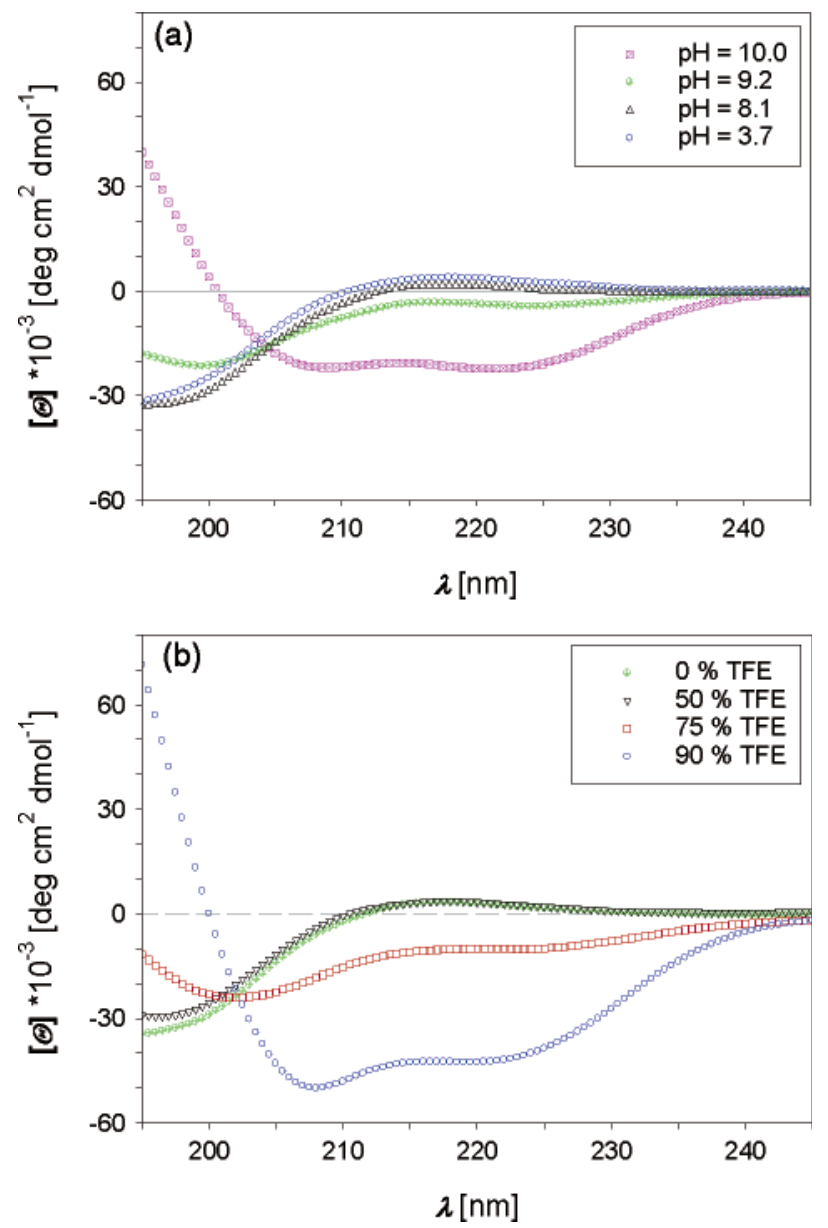

Figure 2. $\mathrm{CD}$ spectra of 30c as a function of $\mathrm{pH}$ (a) and concentration of 2,2,2-trifluoro ethanol (b).

to a dendrimer core is essential for targeting bioproperties as for example antigen-antibody recognition. This motivated us to study the effect of either solvent or length of the peptides on the secondary structure of attached polylysine moieties.

PPDs bearing polylysine chains longer than six amino acid residues were investigated by CD spectroscopy. It is known that 2,2,2-trifluoroethanol (TFE) (29) as well as high $\mathrm{pH}$ (23) induce coil-to-helix transition in polylisine moieties. We employed these protocols to study how the polyphenylene scaffold affects the capability of the attached polypeptides of forming secondary structure. Figure 2 shows the CD spectra of 30c $(c=1.1 \mu \mathrm{mol} / \mathrm{L})$ as a function of $\mathrm{pH}$ (Figure $2 \mathrm{a}$ ) and concentration of TFE (Figure 2b), respectively. The data are representative of all studied compounds $30 \mathbf{a}-\mathbf{c}, 31 \mathbf{a}-\mathbf{c}, 32 \mathbf{a}-\mathbf{c}$, and $33 \mathbf{a}-$ c, and the following conclusions can be drawn.

Below $\mathrm{pH}$ 9, the polypeptide chains form random coils. Above this $\mathrm{pH}$ value, the coils turn into helices. Above $\mathrm{pH} 10$, however, the polypeptides begin to precipitate.

Coil-to-helix transformation can be induced by the exchange of the solvent, namely, when the concentration of TFE is above $75 \%$ vol.

The coil-to-helix transition with TFE as a solvent is induced because the intramolecular repulsion is suppressed $(29-31)$. The same effect can be obtained by the basicity of the aqueous solution being increased (23). The helix content at $\mathrm{pH} 9.9$ and in $90 \%$ TFE was calculated for all polylysine-decorated PPDs according to the method of Chen et al. $(32-34)$. The mean residue ellipticity of a polylysine with $100 \%$ helix content at $222 \mathrm{~nm}$ was taken to be $-37600 \mathrm{deg} \mathrm{cm}^{2} \mathrm{dmol}^{-1}$ (29). The results are
Table 2. Helix Content in Percent from the CD Spectra $(80 \mu \mathrm{g} / \mathrm{mL})$ in Water at $\mathrm{pH}=9.9$ and the Percentage in Comparison with the Measurements in a $90 \%$ TFE Solution

\begin{tabular}{|c|c|c|c|c|c|}
\hline sample & generation & $m_{\text {Lys, UV }}$ & $\mathrm{pH}$ & $\begin{array}{c}\alpha \text {-helix } \\
{[\%]}\end{array}$ & $\begin{array}{c}{[\Theta]_{222}^{\text {water }} /[\Theta]_{222}^{90 \% \mathrm{TFE}}} \\
{[\%]}\end{array}$ \\
\hline $30 a$ & & 9 & 9.9 & 43.6 & 78.2 \\
\hline $30 \mathrm{~b}$ & $n=1$ & 50 & 9.9 & 46.4 & 47.5 \\
\hline $30 \mathrm{c}$ & $x=4$ & 85 & 10 & 60.6 & 53 \\
\hline $31 \mathrm{a}$ & & 9 & 9.9 & 35.2 & 66.8 \\
\hline $31 \mathrm{~b}$ & $n=1$ & 68 & 10.1 & 27.6 & 26.2 \\
\hline $31 \mathrm{c}$ & $x=8$ & 76 & 9.8 & 47 & 45 \\
\hline $32 \mathrm{a}$ & & 6 & 9.9 & 46.4 & 1133 \\
\hline $32 \mathrm{~b}$ & $n=2$ & 28 & 10 & 44.4 & 40.2 \\
\hline $32 \mathrm{c}$ & & 41 & 9.9 & 43.2 & 42.5 \\
\hline $33 \mathrm{a}$ & & 14 & 10 & 24.8 & 22.8 \\
\hline $33 \mathrm{~b}$ & $n=2$ & 41 & 9.9 & 80.6 & 71.2 \\
\hline $33 \mathrm{c}$ & $x=16$ & 89 & 10.1 & 43.3 & 38 \\
\hline
\end{tabular}

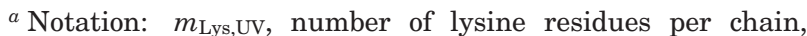
calculated by UV absorption of dendrimers.

summarized in Table 2. For all studied compounds, the helix content at pH 9.9 is about $40 \%$ or above. Furthermore, the helix content determined in $90 \%$ TFE is higher than that determined at $\mathrm{pH}$ 9.9.

The dissociation of polypeptide coiled coils and their organization into single-stranded helices are also evidenced by the ratio of the mean residue ellipticities at 222 and $208 \mathrm{~nm}(33,35)$. Figure 3 a shows the ratio $[\Theta]_{222} /$ $[\Theta]_{208}$ measured at $\mathrm{pH} 9.9$ and in $90 \%$ TFE for all studied compounds. As evident from the plot, for polypeptide chains longer than 10 lysine residues, the ratio $[\Theta]_{222} /$ $[\Theta]_{208}$ remains constant whatever the generation and number of polypeptide chains.

In water the ratio of the mean residue ellipticities at 222 and $208 \mathrm{~nm}$ is nearly one and indicates aggregation between the helices, whereas the value of the measurements in $90 \% \mathrm{TFE}$ is around 0.8 points at dissociation. Shorter chains, comprising less than 10 residues, are less spatially organized and exhibit higher coil content, the latter being evidenced by the minimum in the ellipticity located in the region below $206 \mathrm{~nm}$. An example is presented in Figure $3 \mathrm{~b}$ where the CD spectra of $\mathbf{3 0 a}$ and 30c are compared.

The main conclusion from the CD data is that polypeptides attached on the dendrimers periphery are capable of forming secondary structures. This finding is essential for the design of synthetic multi-immunogens since the secondary structure of epitopes is responsible for the unique antigen-antibody recognition.

3.4.2. UV-Vis Absorption and Fluorescence. The compounds 30a, 31a, 32a, and 33a, each containing a PDI core, were characterized by UV-Vis and fluorescence spectroscopy. As an example, the absorption and emission spectra of 33c, recorded in water and 90\% TFE, are shown in Figure 4. Absorption and emission maxima in aqueous solution are at 580 and $614 \mathrm{~nm}$, respectively, while a small bathochromic shift occurs when changing the solvent from water to $90 \%$ TFE with maxima at 597 (absorption) and $624 \mathrm{~nm}$ (emission).

For all compounds studied, the maxima of absorption are observed at 580-600 nm, being essentially independent of the dendrimer generation and number of peptide residues. Interestingly, the extinction in the less polar solvent water $\left(58000-88000 \mathrm{~cm}^{2} \mathrm{~mol}^{-1}\right)$ is lower than the extinction in TFE solutions (81 000-103000 $\mathrm{cm}^{2}$ $\mathrm{mol}^{-1}$ ). All of the compounds 31-33 exhibit fluorescence. The emission maxima were detected at wavelengths between 615 and $630 \mathrm{~nm}$ upon excitation at $560 \mathrm{~nm}$. The quantum yields in aqueous solutions of all compounds 

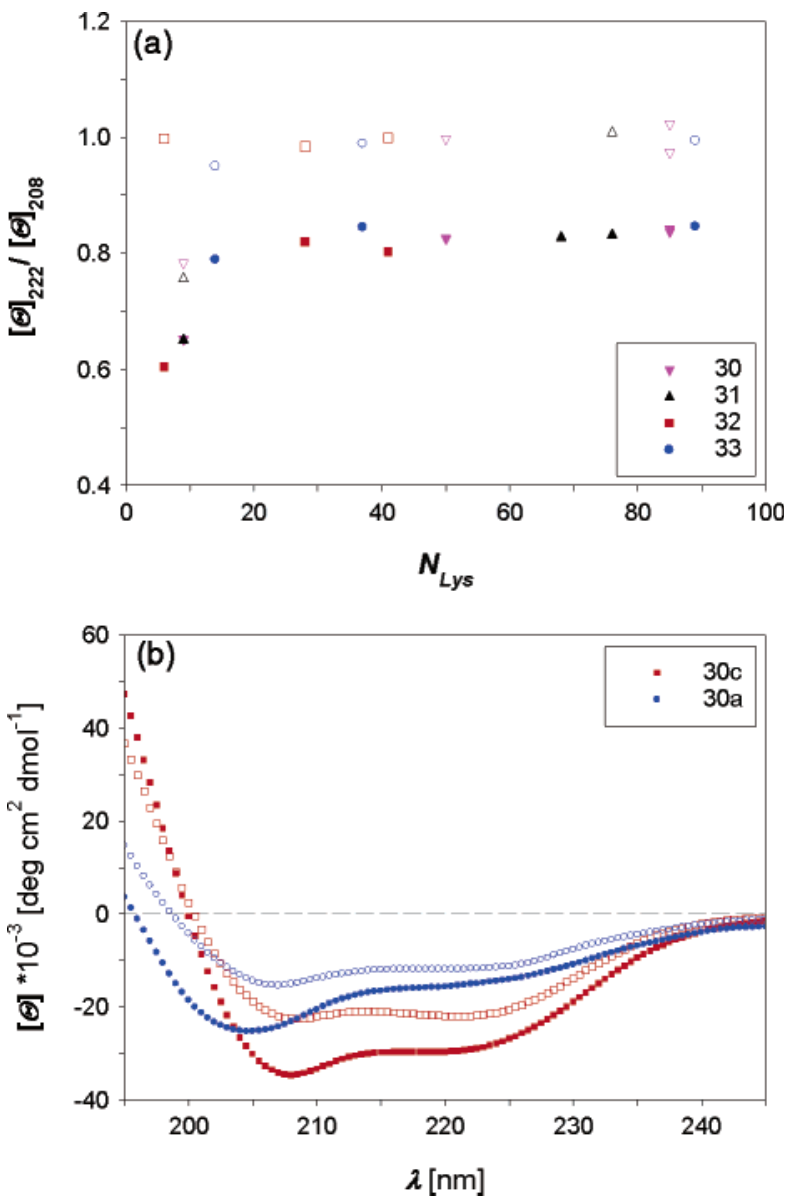

Figure 3. (a) Ratio of the mean residue ellipticities at 222 and $208 \mathrm{~nm}$. $[\Theta]_{222} /[\Theta]_{208}$ of different generations: 30 (red down triangles), 31 (black up triangles) 32 (red squares), 33 (blue circles) in $90 \%$ TFE (filled symbols) and at $\mathrm{pH} 9.9 \pm 0.1$ (empty symbols). (b) CD spectra of 30a and 30c. CD spectra of 30a (circles) and 30c (squares) in $90 \%$ TFE (filled symbols) and at pH 9.9 (empty symbols).

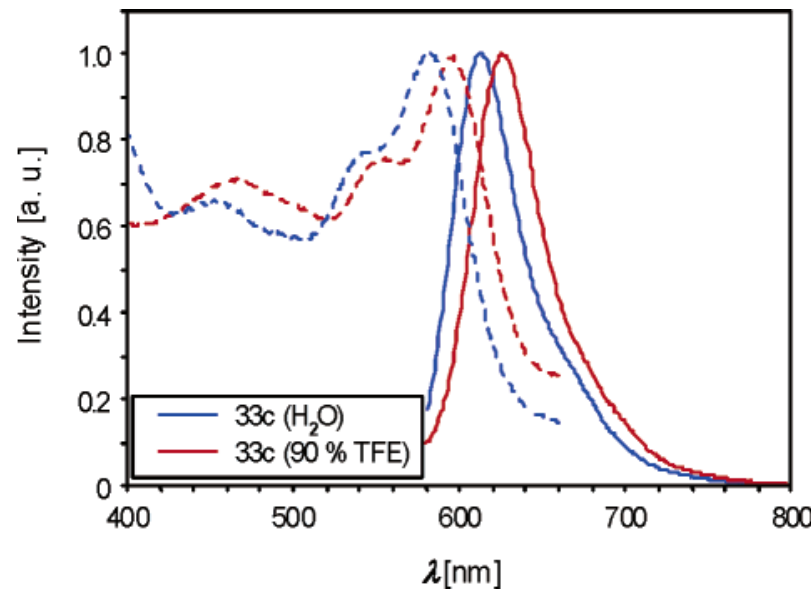

Figure 4. UV-vis absorbance and fluorescence emission spectra of 33c in $90 \%$ TFE and in aqueous solution pH 9.9.

were low (about 1\%). Nevertheless, the optical properties (strong absorption at 580-600 $\mathrm{nm}$ and fluorescence in water) of the peptide-decorated PDI dendrimers allow their identification in complex mixtures of biological samples. These features are very important for in vitro / in vivo studies and pharmaceutically oriented applications since at wavelengths greater than $550 \mathrm{~nm}$ cells and tissue usually do not show any chromophoric properties.

\section{CONCLUSIONS}

We have demonstrated the synthesis of new amino acid functionalized cyclopentadienones. Their Diels-Alder reactions with different core molecules lead to polyphenylene dendrimers functionalized with amino or carboxyl groups. Polylysine fragments were then coupled to these dendrimers employing three different methods.

(i) Grafting of the peptides from the surface of the dendrimer by applying the NCA polymerization strategy. This route affords a decoration of PPDs with polypeptides, connected via their $C$-terminus. Furthermore, the attached polypeptide chains are able to fold into defined secondary structures, an important prerequisite for the preparation of functional bioconjugates.

(ii) Carbodiimide coupling of a protected peptide. The C-terminal carboxyl groups of the peptides are activated employing HBTU/ HOBT and are attached to the surface amino groups of the dendrimers.

(iii) Specific attachment of unprotected peptide fragments under mild conditions via Michael addition of a thiol group of the terminal cysteine of the polypeptide to the maleimide of the PPD. This method allows both $C$ and $N$-terminal attachment of the polypeptide.

The resulting dendrimers have good solubility in water and combine shape-persistence with a controlled number of surface functions. Additionally, when perylenediimide PPDs are used as cores, absorption and fluorescence techniques can be employed for their identification.

By using an asymmetric core molecule and appropriately functionalized cyclopentadienones in a Diels-Alder reaction, we have obtained PPDs bearing two different functional groups. For example, compound 33, a PPD bearing carboxyl and maleimide functions in isolated surface domains, is a scaffold for the creation of a conjugate possessing two different polypetide sequences.

We have recorded circular dichroism (CD) spectra of PPDs bearing different numbers of polypeptide chains of various degrees of polymerization. On the basis of these data, we have concluded that at $\mathrm{pH} 9.9$, as well as in the presence of more than $75 \% \mathrm{TFE}$, the attached polypeptide is capable of forming an $\alpha$-helical secondary structure. This fact is of particular importance for the design of MAP since the secondary structure is essential for the intrinsic properties of its distinct sites. In addition, conjugates synthesized with the perylenediimide core strongly absorb at 580-600 $\mathrm{nm}$ and are fluorescent in water.

Our synthetic methods are not restricted to oligolysines but may be easily adapted to the substitution of PPD with various polypeptide sequences. This opens the way to biofunctonalized compounds with perspectives as spatially defined MAPs with a hydrophilic shell formed of certain epitopes and chromophor-labeled shape-persistent scaffolds.

\section{ACKNOWLEDGMENT}

This work was supported by the Deutsche Forschungsgemeinschaft (SFB 625).

\section{LITERATURE CITED}

(1) Newcome, G. R., Moorefield, C. N., and Vögtle, F. (1996) Dendritic Molecules, VCH Verlagsgesellschaft GmbH, Weinheim.

(2) Bosman, A. W., Janssen, H. M., and Meijer, E. W. (1999) About Dendrimers: Structure, Physical Properties, and Applications. Chem. Rev. 99, 1665-1688.

(3) Fréchet, J. M. J., and Hawker, C. J. (1996) Synthesis and Properties of Dendrimers and Hyperbranched Polymers in 
Comprehensive Polymer Science (S. L. Aggarwal, S. Russo, S, Eds.) 2nd Suppl., Pergamon Press, Oxford.

(4) Stiriba, S.-E., Frey, H., and Haag, R. (2002) Dendritic Polymers in Biological Applications: From Potential to Clinical Use in Diagnostics and Therapy. Angew. Chem., Int. Ed. 41, 1329-1334.

(5) Wiesler, U.-M., Weil, T., and Müllen, K. (2001) Nanosized Polyphenylene Dendrimers. Top. Curr. Chem. 212, 1-40.

(6) Veprek, P., and Jezek, J. (1999) Peptide and glycopeptide dendrimers. Part II. J. Peptide Sci. 5, 203-220.

(7) Tam, J. P. (1996) Recent advances in multiple antigen peptides. J. Immun. Methods 196, 17-32.

(8) Tam, J. P., and Lu, Y.-A. (1989) Vaccine engineering: Enhancement of immunogenicity of synthetic peptide vaccines related to hepatitis in chemically defined models consisting of T- and B-cell epitopes. Biochemistry 86, 9084-9088.

(9) Kohl C., Weil, T., Qu, J., and Müllen K. (2004) Towards Highly Fluorescent and Water-Soluble Perylene Dyes Chem.Eur. J. 10, 5297-5310.

(10) Herrmann, A., Mihov, G., Vandermeulen, G.W. M., Klok, H.-A., and Müllen, K. (2003) Peptide-functionalized polyphenylene dendrimers. Tetrahedron 59, 3925-3935.

(11) Kohn, F., Hofkens, J., Wiesler, U.-M., Cotlet, M., Van der Auweraer, M., Müllen, K., and De Schryver, F. C. (2001) Single-molecule spectroscopy of a dendrimer-based hostguest system. Chem. Eur. J. 7, 4126-4133.

(12) Grimsdale, A. C., Bauer, R., Weil, T., Tchebotareva, N., Wu, J., Watson, M., and Müllen, K. (2002) The Chemical Desymmetrisation of Two- and Three-Dimensional Polyphenylenes as a Key Step to Functional Nanoparticles. Synthesis (Stuttgart) 9, 1229-1239.

(13) Qu, J., Pschirer, N. G., Liu, D., Stefan, A., De Schryver, F. C., and Müllen, K. (2004) Dendronized perylenetetracarboxdiimides with peripheral triphenylamines for intramolecular energy and electron transfer. Chem.-Eur. J. 10, 528-537.

(14) Morgenroth, F., Reuther, E., and Müllen, K. (1997) Polyphenylene dendrimers: From three-dimensional to two- dimensional structures. Angew. Chem., Int. Ed. Engl. 36, 631-634.

(15) Qu, J., Zhang, J., Grimsdale, A. C., and Müllen, K. (2004) Dendronized Perylene Diimide Emitters: Synthesis, Luminescence, and Electron and Energy Transfer Studies. Macromolecules $37,8297-8306$.

(16) Wiesler, U.-M., Berresheim, A. J., Morgenroth, F., Lieser, G., and Müllen, K. (2001) Divergent Synthesis of Polyphenylene Dendrimers: The Role of Core and Branching Reagents upon Size and Shape. Macromolecules 34, 187-199.

(17) Morgenroth, F., Kübel, C., and Müllen, K. (1997) Nanosized polyphenylene dendrimers based upon pentaphenylbenzene units. J. Mater. Chem. 7, 1207-1211.

(18) Morgenroth, F., and Müllen, K. (1997) Dendritic and hyperbranched polyphenylenes via a simple Diels-Alder route. Tetrahedron 53, 15349-15366.

(19) Morgenroth, F., Reuther, E., and Mullen, K. (1997) Polyphenylene Dendrimers: From Three-Dimensional to TwoDimensional Structures. Angew. Chem., Int. Ed. 36, 631634.

(20) Sakai, N., and Ohfune, Y. (1992) Total Synthesis of Galantin I. Acid-Catalyzed Cyclization of Galantinic Acid. J. Am. Chem. Soc. 114, 998-1010.
(21) Ben-Ishai, D., and Berger, A. (1952) Cleavage of NCarbobenzoxy Groups by dry Hydrogen Bromide and Hydrogen Chloride. J. Org. Chem. 17, 1564-1570.

(22) Ram, R. N., and Charles, I. (1997) Selective esterification of aliphatic nonconjugated carboxylic acids in the presence of aromatic or conjugated carboxylic acids catalysed by $\mathrm{NiCl}_{2}$. $6 \mathrm{H}_{2} \mathrm{O}$. Tetrahedron 53, 7335-7340.

(23) Klok, H.-A., Hernandez, J. R., Becker, S., and Mullen, K. (2001) Star-Shaped Fluorescent Polypeptides. J. Polym. Sci. Pol. Chem. 39, 1572-1583.

(24) Poché, D. S., Moore, M. J., and Bowles, J. L. (1999) An Unconventional Method for Purifying the N-Carboxyanhydride Derivates of gamma-Aklyl-L-glutamates. Synth. Commun. 29, 843-854.

(25) Kricheldorf, H. R. (1987) $\alpha$-Amino Acid-N-Carboxyanhydrides and Related Heterocycles, Springer-Verlag, BerlinHeidelberg-New York.

(26) Fields, G. B., and Noble, R. L. (1990) Solid-phase peptide synthesis utilizing 9-fluorenylmethoxycarbonyl amino acids. Int. J. Peptide Res. 35, 161-214.

(27) Choi, J. S., Lee, E. J., Choi, Y. H., Jeong, Y. J., and Park J. S. (1999) Poly(ethylene glycol)-block-poly(L-lysine) Dendrimer: Novel Linear Polymer/Dendrimer Block Copolymer Forming a Spherical Water-Soluble Polyionic Complex with DNA. Bioconjugate Chem. 10, 62-65.

(28) Chaves, F., Calvo, J. C., Carvajal, C., Rivera, Z., Ramirez, L., Pinto, M., Trujillo, M., Guzman, F., and Patarroyo, M. E. (2001) Synthesis, isolation and characterization of Plasmodium falciparum antigenic tetrabranched peptide dendrimers obtained by thiazolidine linkages. J. Peptide Res. 58, 307316.

(29) Maeda, Y., Nakagawa, T., and Kuroda, Y. (2003) Oligopeptide-mediated helix stabilization of model peptides in aqueous solution. J. Peptide Sci. 9, 106-113.

(30) Cammers-Goodwin, A., Allen, T. J., Oslick, S. L., McClure, K. F., Lee, J. H., and Kemp, D. S. (1996) J. Am. Chem. Soc. 118, 3082-3090.

(31) Huang, K., Park, Y. D., Cao, Z. F., and Zhou, H. M. (2001) Reactivation and refolding of rabbit muscle creatine kinase denatured in 2,2,2-trifluoroethanol solutions. Biochim. Biophys. Acta 1545, 305-313.

(32) Chen, Y.-H., Yang, J. T., and Chau, K. H. (1974) Determination of the helix and $\beta$ form of proteins in aqueous solution by circular dichroism. Biochemistry 13, 3350-3359.

(33) Lau, S. Y. M., Taneja, A. K., and Hodges, R. S. (1984) Synthesis of a model protein of defined secondary and quaternary structure. Effect of chain length on the stabilization and formation of two-stranded alpha-helical coiled-coils. J. Biol. Chem. 259, 13253-13261.

(34) Yang J. T., Wu, C.-S., and Martinez, C. H. M. (1986) Calculation of Protein Conformation from Circular Dichroism, Academic Press, New York.

(35) Chitra, R., and Smith, P. E. (2001) Properties of 2,2,2trifluoroethanol and water mixtures. J. Chem. Phys. 114, $426-435$

\section{BC049839K}

